\title{
Convolutive Complex-Valued Independent Component Analysis for Nonlinear Radar Signal Processing and Maritime Weak Target Detection
}

\author{
Hamzeh Ghahramani $\mathbb{D}^{1},{ }^{1}$ Naser Parhizgar $\mathbb{D}^{1},{ }^{1}$ Bijan Abbasi Arand $\mathbb{D}{ }^{2}$ \\ and Morteza Barari ${ }^{3}{ }^{3}$ \\ ${ }^{1}$ Department of Electrical Engineering, Islamic Azad University, Shiraz Branch, Shiraz, Iran \\ ${ }^{2}$ Department of Electrical and Engineering, Tarbiat Modarres University, Tehran, Iran \\ ${ }^{3}$ Department of Electrical and Engineering, ICT Research Institute, Tehran, Iran \\ Correspondence should be addressed to Naser Parhizgar; n.parhizgar47@gmail.com
}

Received 19 May 2021; Revised 27 August 2021; Accepted 19 October 2021; Published 11 November 2021

Academic Editor: Sergio Baselga

Copyright (C) 2021 Hamzeh Ghahramani et al. This is an open access article distributed under the Creative Commons Attribution License, which permits unrestricted use, distribution, and reproduction in any medium, provided the original work is properly cited.

\begin{abstract}
This paper first establishes a new complex independent component analysis (cICA) algorithm based on the spatiotemporal extension of complex-valued entropy bound minimization (CEBM) to separate received complex-valued radar signals. Next, we propose a new cICA-based detector with an open structure to find Swerling model targets, lognormal targets, and sea-surface small floating targets in coherent high-resolution maritime surveillance radars. The detector encountered three major problems when adopting cICA for detection and solved them using three effective suggestions. After performing cICA on the time series received by the radar, we obtained two different sources. Using the first and second theoretical and empirical moment estimates of the K-distribution, the target was selected between these two output source signals. Detector performance was verified quantitatively and qualitatively using the real-life IPIX radar database. Comprehensive experiments on this database with synthetic injected targets showed promising results. The computational time and sample size dependency of the proposed cICA algorithm were also discussed. Finally, a comparison of the detector with several novel detectors for detecting sea-surface floating small targets of the IPIX radar database demonstrated the proposed detector's superiority.
\end{abstract}

\section{Introduction}

The detection of radar targets using the concepts of other fields, e.g., independent component analysis (ICA) [1-3], blind source separation (BSS) $[4,5]$, artificial intelligence, and machine learning methods such as neural networks [6], fuzzy theory [7], graph theory [8], and support vector machines (SVM) [9] has been an active area of research in recent years. These nonlinear strategies have been outlined to overcome troublesome radar detection problems that cannot be resolved via routine radar detection strategies, e.g., Neyman-Pearson (NP)- and Bayesian-based detectors or CFAR and GLRT detector classes. Herein, we propose a new convolutive complex-valued ICA (cICA) algorithm. We utilize it in a new detection scheme to perform such difficult detection problems in maritime high-resolution coherent radars. A highly difficult type of these problems is the detection of sea-surface small floating objects, e.g., small boats, frogman, driftwood, iceberg, and debris. The new detection scheme also performs the detection of Swerling model targets with or without a Doppler frequency embedded in the clutter Doppler cell.

Many other studies have attempted to detect weak targets in maritime high-resolution radars in recent years [10-22]. Some linear methods, e.g., adaptive detection in compound-Gaussian clutter and GLRT-based novel methods [23], are examples of such detectors. The timefrequency analysis (TFA)-based detection method [24] is 
proposed for such scenarios in addition to some nonlinear methods, e.g., fractal-based detection [10-12], time-frequency (TF) feature-based detection [13], other featurebased detection methods [14-19], and polarimetric-feature detection [19]. In coherent high-resolution maritime surveillance radars, due to the complex and nonstationary nature of the returned backscatters from the sea surface, the detection of low-velocity or floating small targets is a challenging problem as the target Doppler frequency occurring in the clutter Doppler cell makes clutter filtering impossible [14]. In this circumstance, the application of nonlinear signal processing strategies, e.g., cICA, is appealing for two primary reasons. First, linear methods, e.g., statistical signal detection theory comprising NP and Bayesian-based algorithms need statistical knowledge of the radar signals (clutter and target) to accurately perform the detection. Second, the performance of novel methods is corrupted with changes in radar signals' statistical characteristics. These algorithms are sensitive to sea state changes and other variables that influence the probability distribution function (PDF) of the radar signals [23]. Moment, target, and clutter are mixed up nonlinearly $[4,6,23]$. Hence, nonlinear signal processing may detect the target from undesirable clutter echoes better [4]. Accordingly, we utilize cICA as a nonlinear signal processing technique to perform radar target detection. We also extend the proposed convolution cICA algorithm to more successfully separate the target from the radar. The proposed detector utilizes the proposed convolutive algorithm to detect radar targets. Finally, the detector's performance is compared with several detectors recently proposed within the recognized IPIX radar database [25], thereby illustrating its superiority.

ICA, the most attractive solution for the BSS problem, yields a useful decomposition with two simple ambiguities, scaling and permutation, under the assumption of statistical independence among source signals [26-28]. It separates $N$ source signals from $N$ mixtures (resulting from the mixing of the sources) recorded at $N$ existing sensors, e.g., $N$ microphone in an acoustic system or $N$ antennas in a communication or radar system. There are two classes of ICA algorithms: instantaneous and convolutive. While instantaneous ICA does not apply to most practical signal processing problems, convolutive ICA algorithms, e.g., spatiotemporal FastICA (STFICA) [29] fit several important practical scenarios such as multitalker speech separation and cochannel interference mitigation in multi-input multioutput wireless communication systems [28]. Besides, complex-valued ICA (cICA) is widely utilized in several applications, including radar, communications, and biomedicine [28]. To the best of our knowledge, convolutive cICA has not been widely studied yet. Complex-values STFICA (CSTFICA) is one of the few methods of this group and can be utilized for maritime radar target detection [4]. Since we consider radar received time series at a cell under test (CUT) or each reference cell (RC) as a mixture, cICA algorithms can be used to demix it to attain its containing sources. Assume that the CUT contains the target, and we consider it as a mixture. It is known that all range cells contain sea clutter, including the CUT and reference cells
(RCs). Thus, if we apply a cICA algorithm to the mixture, we can obtain two latent sources, i.e., clutter and target. One of these sources will be the desired target signal that must be selected based on a detection decision threshold. In addition, due to the complex nature of radar backscatter echoes from the sea surface and targets, the complex-valued radar signal mixtures received at the CUT and RCs have a convolutive nature. Therefore, an effective convolutive cICA algorithm should be developed to separate targets in practical scenarios. Accordingly, we propose a new convolutive cICA algorithm to separate the target from the clutter in coherent radars using a spatiotemporal extension of CEBM [1].

In general, one can achieve cICA via four types of diversity: non-Gaussianity, nonwhiteness, noncircularity, and nonstationarity or a combination of them [30]. NonGaussianity is the most widely used diversity. Most cICA algorithms overlook sample dependence, whereas some of them incorporate noncircularity by utilizing second-order statistics. Some algorithms employ two types of diversity, i.e., non-Gaussianity and nonwhiteness. CEBM [1], noncircular FastICA [31], and the complex fixed-point algorithm (CFPA) [32] utilize both non-Gaussianity and noncircularity to perform separation tasks. Complex-valued entropy rate bound minimization (CERBM) [30] exploits all three important types of diversity: non-Gaussianity, sample dependence, and noncircularity. Besides, the cICA algorithms assume that the number of sources and mixtures (sensors) is the same, while there is just one mixture in the monostatic or bistatic radars (since they have only one antenna or one radar received signal). If we expect that the radar signal contains at least two sources, i.e., target and clutter, we require at least two mixtures or two received signals at antennas. This need for mixture is overcome through the proposed detector utilizing the clutter samples of one single frame of the recorded signal at RCs. In fact, the proposed detector suppresses the clutter using the clutter itself. Other general assumptions of the cICA algorithms are valid for radar signals since, as mentioned in [4], clutter and target signals are independent of each other. They are also noncircular and non-Gaussian distributed signals.

This paper attempts to design a cICA-based radar detector and a convolutive cICA algorithm to solve challenging detection problems, e.g., sea-surface small floating target detection and very low signal-to-clutter ratio (SCR) Swerling targets. The main contributions of this paper are as follows:

(1) We exploit cICA algorithms for radar signal separation and target detection. We design a new convolutive cICA algorithm because there is no effective algorithm in the cICA literature to separate radar signals in real-life scenarios.

(2) We adopt the cICA to design a new radar detector. ICA was initially designed to perform speech separation, but over the years, complex-valued ICA demonstrated its potential in other fields, e.g., biomedicine and communication. To the best of our knowledge, despite the potential of cICA, it is not widely applied for radar signal processing. Herein, we utilize it as a tool for radar target detection. Three 
main challenges arise when designing a cICA-based detector. We solve these problems using three effective suggestions in three distinct blocks of the detector. We prove that the cICA-based detector is well capable of separating real-life radar signals for target detection. Our experiments on the real-life IPIX radar database support the validity of our claim.

The remainder of this paper is organized as follows. In Section 2, we propose a new convolutive cICA algorithm. The cICA-based detector is proposed in Section 3. Through some simulations, utilizing synthetic Swerling and lognormal targets and real-life IPIX radar data, the validity and effectiveness of the proposed cICA-based detector when using the proposed cICA algorithm are demonstrated in Section 4 by comparing with several novel detectors. This section also introduces the detector's performance when utilizing some other cICA algorithm, as well as its computational time and sample dependency. Finally, Section 5 concludes the paper.

\section{Proposed Convolutive Complex-Valued Independent Component Analysis (cICA) Algorithm}

To explain the complex-valued ICA algorithms, assume that $\mathbf{z}(t)=\mathbf{z}_{R}(t)+j \mathbf{z}_{I}(t)$ is a complex random variable with zero mean, where $\mathbf{z}_{R}(t)$ and $\mathbf{z}_{I}(t)$ denote the real and imaginary parts, respectively $(j=\sqrt{-1}$ is the imaginary unit). Furthermore, $\mathbf{s}(t)=\left[s_{1}(t), \ldots, s_{N}(t)\right]^{T} \in \mathbb{C}^{N}$ are the complexvalued sources mixed through an $N \times N$ nonsingular mixing matrix A. Complex-valued mixtures are obtained as $\mathbf{x}(t)=$ $\left[\begin{array}{lll}x_{1}(t) & \cdots & x_{N}(t)\end{array}\right]^{T}=\mathbf{A s}(t)$ and $\mathbf{x}(t) \in \mathbb{C}^{N}$, where $x(t)=$ $x_{R}(t)+j x_{I}(t)$ is a complex-valued mixture signal. The sources are separated through $\mathbf{z}(t)=\mathbf{W} \mathbf{x}(t)$, where $\mathbf{z}(t)=$ $\left[z_{1}(t), \ldots, z_{N}(t)\right]^{T} \in \mathbb{C}^{N}$ is an estimation of $\mathbf{s}(t)$ and $z(t)=$ $z_{R}(t)+j z_{I}(t)$ is a complex-valued source signal and is known as demixing or separation matrix. In CICA, the observed data (here the radar received signal), $x_{m}(t)$, are typically expressed as a linear combination of latent sources (here target, clutter, noise, jamming, and so on), such that the instantaneous cICA model can be formulated in the standard form as follows:

$$
x_{m}(t)=\sum_{n=1}^{N} a_{n m}^{*} s_{n}(t)=\mathbf{a}_{m}^{H} \mathbf{s}(t), \quad 1 \leq m \leq N .
$$

In signal processing notation, $N$ denotes the number of mixture which is equal to the number of mixtures, $x_{m}(t) \in \mathbb{C}^{T}$ is called the $m$ th complex mixture, $s_{n}(t) \in \mathbb{C}^{T}$ is the $n$th latent complex source for $1 \leq n \leq N$, and $a_{n m}$ are the complex coefficients of the mixing system expressed by the $N \times N \quad$ mixing matrix $\quad \mathbf{A}=\left[\mathbf{a}_{1}, \ldots, \mathbf{a}_{N}\right]^{H}$ and $\mathbf{a}_{m}$ $=\left[a_{1 m}, \ldots, a_{n m}\right]^{T}$.

The complex-valued convolutive mixing model is formulated as $\mathbf{x}(t)=\underline{\mathbf{A}} \underline{\mathbf{s}}(t)$, where $\underline{\mathbf{A}} \in \mathbb{C}^{N \times N \times L}$ and $\underline{\mathbf{s}}(t) \in \mathbb{C}^{N \times T \times L}$ are the sources and their delayed attenuated versions, respectively. The estimation of complex-valued separated sources is $\mathbf{z}(t)=\underline{\mathbf{W}} \mathbf{x}(t)$, where
$\underline{\mathbf{W}}=\left[\underline{\mathbf{w}}_{1}, \ldots, \underline{\mathbf{w}}_{N}\right]^{H} \in \mathbb{C}^{N \times N \times L}$, and the mixing model for one single complex-valued mixture is as follows:

$$
x_{m}(t)=\sum_{l=0}^{L-1} \sum_{n=1}^{N} \underline{a}_{m n l}^{*} s_{n}(t-l), \quad \text { for } 1 \leq m \leq N,
$$

where $L$ is a filter length and each $\underline{a}_{m n l}$ (an element of $\underline{\mathbf{A}}$ ) is a complex coefficient that attenuates the delayed version $(l$ sample) of $s_{n}(t)$. All the attenuated and delayed versions of $s_{n}(t)$ are summed to construct the convolutive mixture $x_{m}(t)$. To invert the convolutive mixtures of equation (2), a set of similar FIR filters is typically used as follows:

$$
z_{n}(t)=\sum_{l=0}^{L} \sum_{j=1}^{N} \underline{w}_{n m l} x_{m}(t-l), \quad 1 \leq n \leq N .
$$

If we assume that there are two sources in the radar received signal at the CUT, i.e., clutter $C_{\text {CUT }}(t)$ and target $T g_{\text {CUT }}(t)$, the estimated source signals $z_{1}(t)$ and $z_{2}(t)$ using this separating system would be the estimations of the source signals $s_{1}(t)=$ $C_{\mathrm{CUT}}(t)$ and $s_{2}(t)=T g_{\mathrm{CUT}}(t)$ at discrete time $t$. Note that the complex mixtures vector $\mathbf{x}(t)$ is prewhitened spatially and temporally to obtain $\underline{\mathbf{v}}(t)=\left[\begin{array}{llll}\underline{v}_{1}(t) & \cdots & \underline{v}_{N}(t)\end{array}\right]^{T}=\underline{\mathscr{B}} \mathbf{x}(t)$ where $\mathscr{B}$ is the whitening filter and

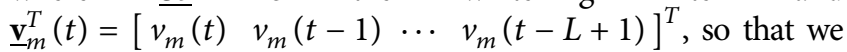
have $E\left(v_{n}\left(t_{k}\right) v_{m}\left(t_{l}\right)\right)=\delta_{n m} \delta_{t_{k} t_{l}}$. For the whitening stage, we adopted a method similar to that in [33].

We utilized various ICA algorithms through the proposed detector. The results of our experiments on the IPIX radar database showed that the convolutive methods can separate the target from the clutter signal. In addition, CERBM is the only known instantaneous cICA algorithm capable of radar signal separation. Overall, an instantaneous cICA, a convolutive real-valued ICA, and a convolutive cICA algorithm can separate target from clutter: CERBM, STFICA, and the proposed CCEBM, respectively. The other mentioned real-valued ICA and cICA algorithms fail to perform the detection via the proposed detector. It means that the most important diversities for separating radar signals are noncircularity, nonwhiteness, and non-Gaussianity, which should be used simultaneously to perform the separation. According to our experiments, the convolutional ICA algorithm is more likely to perform the separation stage in the detection of radar signals, as proven by STFICA. Since STFICA is utilized to separate a coherent complex-valued signal with in-phase $(I)$ and quadrature $(Q)$ components, we named it IQSTFICA in this paper. Two types of diversity are used in STFICA and the proposed CCEBM, non-Gaussianity and convolutedness, which impacted on effective separation. In summary, the use of noncircularity and non-Gaussianity in the convolution model of the cICA results in the best separation performance in the field of complex-valued radar signal processing. To the best of our knowledge, a few convolutive cICA algorithms [32] so far been introduced. Therefore, in this section, we present a new convolutive cICA algorithm to separate radar signals in practical scenarios via our proposed detector after reviewing the first two algorithms. 
2.1. Complex-Valued Entropy Bound Minimization (CEBM). CEBM takes both non-Gaussianity and noncircularity into account. By proposing a new (differential) flexible entropy estimator for a complex random variable based on the principle of maximum entropy, the CEBM is a complex ICA algorithm by entropy bound minimization that adopts a line search optimization procedure using a projected conjugate gradient. In this study, noncircularity means that the signal is not second-order circular. A complex-valued separated signal, i.e., $z(t)$, is second-order circular if $E\left(z^{2}\right)=0$. In addition, the mutual information $\mathscr{I}_{r}\left(z_{1} ; \ldots ; z_{N}\right)$ among $N$ random variable $z_{n}(t), n=1, \ldots, N$, is a natural cost function used for separation of $N$ independent source signals as follows:

$$
\mathscr{I}\left(z_{1} ; \ldots ; z_{N}\right)=\sum_{n=1}^{N} H\left(z_{n}\right)-2 \log |\operatorname{det}(\mathbf{W})|-H(\mathbf{x}),
$$

where $H(\cdot)$ denotes the entropy. Note that the time index $t$ is omitted for simplicity in this equation and the subsequent discussion. Since the mixtures are prewhitened and the demixing matrix is constrained to be a unitary matrix, in unitary ICA approaches, we have $|\operatorname{det}(\mathbf{W})|=1$, and the cost function is as follows:

$$
\mathscr{I}\left(z_{1} ; \ldots ; z_{N}\right)=\sum_{n=1}^{N} H\left(z_{n}\right)-H(\mathbf{x}),
$$

where $H(\mathbf{x})$ is a constant with respect to $\mathbf{W}$; therefore, we can use $\mathscr{I}\left(z_{1} ; \ldots ; z_{N}\right)=\sum_{n=1}^{N} H\left(z_{n}\right)$ as a cost function.

To explain the CEBM, consider the following decomposition:

$$
\left[z_{R}, z_{I}\right]^{T}=\mathbf{B}[u, v]^{T}=\mathbf{B} r[\cos \theta, \sin \theta]^{T},
$$

where $\mathbf{B}$ is a $2 \times 2$ nonsingular matrix, $[u, v]^{T}$ are a pair of random variables of zero mean, and $r$ and $\theta$ are the complex magnitude (modulus) and the principal value of the argument of $u+j v$, respectively. Using this decomposition, one can obtain two upper bounds for $H(z)$ as follows:

$$
\begin{aligned}
H(z)= & \log |\operatorname{det}(\mathbf{B})|+H(u, v) \\
& \leq \log |\operatorname{det}(\mathbf{B})|+H(u)+H(v) \\
= & H^{[\operatorname{bound}, \mathrm{I}]}(z, \mathbf{B}), \\
H(z)= & \log |\operatorname{det}(\mathbf{B})|+H(u, v) \\
= & \log |\operatorname{det}(\mathbf{B})|+E(\log r)+H(r, \theta) \\
& \leq \log |\operatorname{det}(\mathbf{B})|+E(\log r)+H(r)+H(\theta) \\
& \leq \log |\operatorname{det}(\mathbf{B})|+E(\log r)+H(r)+\log (2 \pi) \\
= & H^{[\operatorname{bound}, \mathrm{II}]}(z, \mathbf{B}) .
\end{aligned}
$$

By QR-decomposition of the matrix $\mathbf{B}=\mathbf{Q R}$ and assuming $\mathbf{Q}=\mathbf{I}$ and $\mathbf{B}=\mathbf{R}$ (since $\mathbf{Q}$ can be absorbed by the demixing vector $\mathbf{w})$, we have $\left[z_{R}, z_{I}\right]^{T}=\mathbf{R}[u, v]^{T}$ and

$$
E\left(\left[\begin{array}{c}
z_{R} \\
z_{I}
\end{array}\right]\left[\begin{array}{ll}
z_{R} & z_{I}
\end{array}\right]\right)=\left[\begin{array}{cc}
\sigma_{R}^{2} & \rho \\
\rho & \sigma_{I}^{2}
\end{array}\right]=\mathbf{R R}^{T}
$$

Since $\mathbf{R}$ is a lower triangular matrix and the covariance matrix is a symmetric and positive definite matrix, the product $\mathbf{R R}^{T}$ defines a Cholesky factorization. After solving equation (8), one can obtain

$$
\mathbf{R}^{-1}=\left[\begin{array}{cc}
\frac{1}{\sigma_{R}} & 0 \\
-\frac{\rho}{\sigma_{R} \sqrt{\Delta_{1}}} & \frac{\sigma_{R}}{\sqrt{\Delta_{1}}}
\end{array}\right],
$$

where $\Delta_{1}=\sigma_{R}^{2} \sigma_{I}^{2}-\rho^{2}$, and the explicit expression for $u, v$, and $r$ can readily be obtained as

$$
\begin{aligned}
& u=\frac{z_{R}}{\sigma_{R}}, \\
& v=-\frac{\rho z_{R}}{\sigma_{R} \sqrt{\Delta_{1}}}+\frac{\sigma_{R} z_{I}}{\sqrt{\Delta_{1}}}, \\
& r=\sqrt{u^{2}+v^{2}}=\sqrt{\frac{\Delta_{2}}{\Delta_{1}}},
\end{aligned}
$$

where $\Delta_{2}=z_{R}^{2} \sigma_{I}^{2}+z_{I}^{2} \sigma_{I}^{2}-2 z_{R} z_{I} \rho$. Hence, the entropy estimator is obtained as

$$
\begin{aligned}
H_{k_{1}, k_{2}}^{[\text {bound,I] }}(z)= & 0.5 \log \left(\Delta_{1}\right)+\log (2 \pi e) \\
& -V_{k_{1}}^{[\mathrm{I}]}\left\{E\left[G_{k_{1}}^{[\mathrm{I}]}(u)\right]\right\}-V_{k_{2}}^{[\mathrm{I}]}\left\{E\left[G_{k_{2}}^{[\mathrm{I}]}(v)\right]\right\}, \\
H_{k}^{[\text {bound,II] }}(z)= & 0.5 \log \left(\Delta_{1}\right)+\log (2 \pi e) \\
& -V_{k}^{[\mathrm{II}]}\left\{E\left[G_{k}^{[\mathrm{II}]}(r)\right]\right\}, \\
\widehat{H}(z)= & \min \left(\min _{1 \leq k_{1}, k_{2} \leq K^{[\mathrm{II}]}}\left(H_{k_{1}, k_{2}}^{[\mathrm{bound}, \mathrm{I}]}(z)\right), \min _{1 \leq k \leq K^{[\mathrm{II}]}}\left(H_{k}^{[\text {bound,II }]}(z)\right)\right),
\end{aligned}
$$

where $G_{k_{1}}^{[\mathrm{I}]}(\cdot), \quad G_{k_{2}}^{[\mathrm{I}]}(\cdot), \quad 1 \leq k_{1}, k_{2} \leq K^{[\mathrm{I}]}, \quad$ and $G_{k}^{[\mathrm{II}]}(\cdot)$, $1 \leq k \leq K^{[\mathrm{II}]}$, are the measuring functions and $V_{k_{1}}^{[\mathrm{I}]}\left\{E\left[G_{k_{1}}^{[\mathrm{I}]}(u)\right]\right\}, V_{k_{2}}^{[\mathrm{I}]}\left\{E\left[G_{k_{2}}^{[\mathrm{I}]}(v)\right]\right\}$, and $V_{k}^{[\mathrm{II}]}\left\{E\left[G_{k}^{[\mathrm{II}]}(r)\right]\right\}$ are the negentropies of $u, v$, and $r$ that are directly used from [34]. This entropy estimator uses all the second-order statistical information of a complex random variable.

The measuring function is introduced in Table 1 [1]. To estimate the entropy bound of equation (11), there are $\left(K^{[\mathrm{I}]}\right)^{2}$ entropy estimators of type I and $K^{[\mathrm{II}]}$ entropy estimators of type II that the algorithm chooses the tightest one as the entropy estimator.

To derive the updates of the CEBM, reference [1] divides the problem of $\mathscr{I}\left(z_{1} ; \ldots ; z_{N}\right)$ minimization with respect to $\mathbf{W}=\left[\begin{array}{lll}\mathbf{w}_{1} & \cdots & \mathbf{w}_{N}\end{array}\right]^{H}$ into a series of subproblems such that $\mathscr{I}\left(z_{1} ; \ldots ; z_{N}\right)$ is minimized with respect to each row vector $\mathbf{w}_{n}, n=1, \ldots, N$. The algorithm first updates $\mathbf{w}_{n}$, while $\mathbf{w}_{i}$, $i=1, \ldots, n-1, n+1, \ldots, N$, are kept constant. In the following, we can rewrite $\operatorname{det}|\mathbf{W}|$ as follows [1]:

$$
\operatorname{det}|\mathbf{W}|=\left|S_{n}^{H} \mathbf{w}_{n}\right|
$$

where $S=\sqrt{\left|\operatorname{det}\left(\mathbf{W}_{n} \mathbf{W}_{n}^{H}\right)\right|}, \mathbf{W}_{n}$ is an $(N-1) \times N$ matrix obtained by removing the $n$th row vector of $\mathbf{W}$, 
$\mathbf{W}_{n}=\left[\mathbf{w}_{1}, \ldots, \mathbf{w}_{n-1}, \mathbf{w}_{n+1}, \ldots, \mathbf{w}_{N}\right]^{H}$, and $\mathbf{h}_{n}$ is a vector of unit length satisfying $\mathbf{W}_{n} \mathbf{h}_{n}=0$. In [1], a strategy for calculation of $\mathbf{h}_{n}$ is presented. All the row vectors of $\mathbf{W}$ have unit variance, i.e., $\left\|\mathbf{w}_{n}\right\|=1$, and the mixtures are prewhitened, implying that the sources are of unit variance. It also removes the inherent permutation and scaling ambiguity of the ICA solution. Finally, the method solves the following constraint optimization problem based on the cost function (2) and using equations (11) and (12):

$$
\begin{aligned}
\min J_{n}\left(\mathbf{w}_{n}\right) & =\widehat{H}\left(z_{n}\right)-2 \log \left|\mathbf{h}_{n}^{H} \mathbf{w}_{n}\right|+C \\
\text { s.t. }\left\|\mathbf{w}_{n}\right\| & =1,
\end{aligned}
$$

where $J_{n}\left(\mathbf{w}_{n}\right)$ is a cost function representing mutual information, $z_{n}=\mathbf{w}_{n}^{H} \mathbf{x}$, and $C$ is a quantity independent of $\mathbf{w}_{n}$. This problem can be written as $L_{n}\left(\mathbf{w}_{n}, \lambda\right)=J_{n}\left(\mathbf{w}_{n}\right)+\lambda\left(\mathbf{w}_{n}^{H} \mathbf{w}_{n}-1\right)$ using the Lagrangian multiplier. The conjugate gradients using Wirtinger derivatives are

$$
\begin{gathered}
\frac{\partial J_{n}\left(\mathbf{w}_{n}\right)}{\partial \mathbf{w}_{n}^{*}}=\frac{\partial \widehat{H}\left(z_{n}\right)}{\partial \mathbf{w}_{n}^{*}}-\frac{\mathbf{h}_{n}}{\mathbf{w}_{n}^{H} \mathbf{h}_{n}}, \\
\frac{\partial L_{n}\left(\mathbf{w}_{n}, \lambda\right)}{\partial \mathbf{w}_{n}^{*}}=\frac{\partial J_{n}\left(\mathbf{w}_{n}\right)}{\partial \mathbf{w}_{n}^{*}}+\lambda \mathbf{w}_{n},
\end{gathered}
$$

where the Lagrangian multiplier $\lambda$ is a real number, superscript ${ }^{*}$ denotes conjugation, and the explicit expression for $\left(\partial \hat{H}\left(z_{n}\right) / \partial \mathbf{w}_{n}^{*}\right)$ and $\left(\partial L_{n}\left(\mathbf{w}_{n}, \lambda\right) / \partial \mathbf{w}_{n}^{*}\right)$ can be found in [1]. After removing the term strictly collinear with $\mathbf{w}_{n}[1]$, the projected conjugate gradient on the constraint surface $\left\|\mathbf{w}_{n}\right\|=1$ is as follows:

$$
\begin{aligned}
& \mathbf{u}_{n}^{+}=\frac{\partial J_{n}\left(\mathbf{w}_{n}\right)}{\partial \mathbf{w}_{n}^{*}}-\operatorname{Re}\left\{\mathbf{w}_{n}^{H} \frac{\partial J_{n}\left(\mathbf{w}_{n}\right)}{\partial \mathbf{w}_{n}^{*}}\right\} \mathbf{w}_{n}, \\
& \mathbf{u}_{n}=\frac{\mathbf{u}_{n}^{+}}{\left\|\mathbf{u}_{n}^{+}\right\|},
\end{aligned}
$$

where the projection conjugate gradient is calculated using the method proposed in [1]. $\mathbf{u}_{n}$ is a vector of unit length, orthogonal to the previous $\mathbf{w}_{n}$. It points to the steepest ascent direction. Therefore, the line search algorithm for CEBM is obtained as follows:

$$
\begin{aligned}
\mathbf{w}_{n}^{+} & =\mathbf{w}_{n}-\mu \mathbf{u}_{n}, \\
\mathbf{w}_{n}^{[\text {new }]} & =\frac{\mathbf{w}_{n}^{+}}{\left\|\mathbf{w}_{n}^{+}\right\|},
\end{aligned}
$$

where $\mu>0$ is the real-valued step size. This line search procedure is repeated over different row vectors of $\mathbf{W}$ until convergence. The main steps of CEBM are as follows [1]:

(i) For $n=1, \ldots, N$, do the following:

(1) Calculate vector $\mathbf{h}_{n}$, which is orthogonal to $\mathbf{w}_{i}$, $i=1, \ldots, n-1, n+1, \ldots, N$.

(2) Let $z_{n}=\mathbf{w}_{n}^{H} \mathbf{x}$, and estimate the entropy of $z_{n}$ by using the estimator given in (11). Note that the tightest estimates of the entropies are used as the entropy estimate.

(3) Calculate the conjugate gradient $\left(\partial J_{n}\left(\mathbf{w}_{n}\right) / \partial \mathbf{w}_{n}^{*}\right)$ by using (14), considering the selected entropy bound.

(4) Calculate the projected conjugate gradient of (15).

(5) Update $\mathbf{w}_{n}$ based on (16).

Since the CEBM algorithm is designed for instantaneous mixtures, it is unable to separate radar signal mixtures because of its convolutive nature as we observed in our experiments. Therefore, we modify the CEBM algorithm to separate the convolutive mixtures.

2.2. Proposed Convolutive CEBM (CCEBM). The extension of the CEBM to the convolutive case, which we call it convolutive CEBM (CCEBM), is similar to the extension of FastICA to STFICA in [29]. In this case, the complex-valued demixing coefficients are constrained to be paraunitary as in CSTFICA [4]. Here, we can use both sequential and symmetric orthogonalizations to impose paraunitary constraints on complex coefficients simply by replacing the transpose operators by Hermitian operators in these two STFICA approaches. Therefore, our proposed CCEBM algorithm with sequential orthogonalization is as follows:

(1) Convolutive mixtures $\mathbf{x}(t)$ are prewhitened spatially and temporally to obtain $\underline{\mathbf{v}}(t)$.

(2) Compute the $n$th separated source for $1 \leq t \leq T$ as follows:

$$
z_{n}(t)=\sum_{l=0}^{\widehat{L}-1} \sum_{m=1}^{N} \underline{w}_{n m l}^{*} v_{m}(t-l)=\underline{\mathbf{w}}_{n}^{H} \underline{\mathbf{v}}(t), \quad \text { for } 1 \leq n \leq N
$$

where $L$ is a filter length and

$$
\begin{aligned}
\underline{\mathbf{w}}_{n} & =\left[\begin{array}{llll}
\underline{\mathbf{w}}_{n 1}^{H} & \underline{\mathbf{w}}_{n 2}^{H} & \cdots & \underline{\mathbf{w}}_{n N}^{H}
\end{array}\right]^{H}, \\
\underline{\mathbf{w}}_{n m} & =\left[\begin{array}{llll}
\underline{w}_{n m 0} & \underline{w}_{n m 1} & \cdots & \underline{w}_{n m(\widehat{L}-1)}
\end{array}\right]^{H} .
\end{aligned}
$$

For $n=1, \ldots, N$, do the following:

(3) Estimate the entropy of $z_{n}$ via equation (11). Among all possible entropies, the tightest one is used as the estimated entropy bound.

(4) Calculate vector $\underline{\mathbf{h}}_{n}$, which is orthogonal to $\underline{\mathbf{w}}_{i}$, $i=1, \ldots, n-1, n+1, \ldots, N$, and satisfies $\operatorname{det}|\underline{\mathbf{W}}|=$ $\left|S \underline{\mathbf{h}}_{n}^{H} \underline{\mathbf{w}}_{n}\right|$, where $\underline{\mathbf{h}}_{n}$ is calculated similarly to $\overline{\mathbf{h}}_{n}$ in (12).

(5) Calculate the conjugate gradient $\left(\partial J_{n}\left(\underline{\mathbf{w}}_{n}\right) / \partial \underline{\mathbf{w}}_{n}^{*}\right)$, depending on the selected entropy bound by using the following equation:

$$
\begin{gathered}
\frac{\partial J_{n}\left(\underline{\mathbf{w}}_{n}\right)}{\partial \underline{\mathbf{w}}_{n}^{*}}=\frac{\partial \hat{H}\left(z_{n}\right)}{\partial \underline{\mathbf{w}}_{n}^{*}}-\frac{\underline{\mathbf{h}}_{n}}{\underline{\mathbf{w}}_{n}^{H} \underline{\mathbf{h}}_{n}}, \\
\frac{\partial L_{n}\left(\underline{\mathbf{w}}_{n}, \lambda\right)}{\partial \underline{\mathbf{w}}_{n}^{*}}=\frac{\partial J_{n}\left(\underline{\mathbf{w}}_{n}\right)}{\partial \underline{\mathbf{w}}_{n}^{*}}+\lambda \underline{\mathbf{w}}_{n},
\end{gathered}
$$


where $\left(\partial \hat{H}\left(z_{n}\right) / \partial \underline{\mathbf{w}}_{n}^{*}\right)$ is calculated similarly to (14).

(6) Calculate the projected conjugate gradient as follows:

$$
\underline{\mathbf{u}}_{n}^{+}=\frac{\partial J_{n}\left(\underline{\mathbf{w}}_{n}\right)}{\partial \underline{\mathbf{w}}_{n}^{*}}-\operatorname{Re}\left\{\underline{\mathbf{w}}_{n}^{H} \frac{\partial J_{n}\left(\underline{\mathbf{w}}_{n}\right)}{\partial \underline{\mathbf{w}}_{n}^{*}}\right\} \underline{\mathbf{w}}_{n}, \underline{\mathbf{u}}_{n}=\frac{\underline{\mathbf{u}}_{n}^{+}}{\left\|\underline{\mathbf{u}}_{n}^{+}\right\|} .
$$

(7) Update $\underline{\mathbf{w}}_{n}$ as $\underline{\mathbf{w}}_{n} \leftarrow \underline{\mathbf{w}}_{n}-\mu \underline{\mathbf{u}}_{n}$.

(8) Normalize the length of $\underline{\mathbf{w}}_{n}$ as follows:

$$
\underline{\mathbf{w}}_{n} \leftarrow \frac{\underline{\mathbf{w}}_{n}}{\sqrt{\underline{\mathbf{w}}_{n}^{H} \underline{\mathbf{w}}_{n}}}
$$

(9) While $\underline{\mathbf{w}}_{n}$ is not paraunitary with $\underline{\mathbf{w}}_{1}, \ldots, \underline{\mathbf{w}}_{n-1}$,

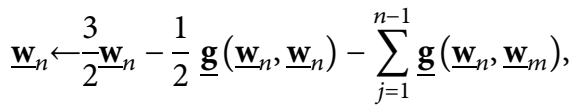

where

$$
\begin{aligned}
\underline{\mathbf{g}}\left(\underline{\mathbf{w}}_{n}, \underline{\mathbf{w}}_{m}\right) & =\left[\begin{array}{llll}
\underline{\mathbf{g}}_{n m 1}^{H} & \underline{\mathbf{g}}_{n m 2}^{H} & \cdots & \underline{\mathbf{g}}_{n m N}^{H}
\end{array}\right]^{H}, \\
\underline{\mathbf{g}}_{n m k} & =\underline{\mathbf{C}}_{n m \underline{\mathbf{w}}_{m k},}
\end{aligned}
$$

and the $(p, q)$ th element of $\underline{\mathbf{C}}_{n j}$ is given by

$\left[\underline{\mathbf{C}}_{n m}\right]_{p q}=\left\{\sum_{k=1}^{N} \sum_{l=0}^{\widehat{L}-1} \underline{w}_{m k l}^{*} \underline{w}_{n k(l+p-q)}\right.$, if $|p-q|<\frac{\widehat{L}-1}{2}, 0$, otherwise.

Symmetric orthogonalization can be extracted similarly [29]. Note that the demixing matrix $\mathbf{W}$ can be initialized randomly or using the equation $\mathbf{W}=$ $\operatorname{Kron}\left(\mathbf{I}_{N \times N}, \mathbf{B}\right), \mathbf{B}=\left[0_{[(L-1) / 2]} \times 1^{T}, 1,0_{[(L-1) / 2] \times 1}^{T}\right]^{T}$, where Kron returns the Kronecker tensor product of matrices $\mathbf{I}$ and $\mathbf{B}$. Since $\mathbf{I}$ is an $N$-by- $N$ matrix and $\mathbf{B}$ is a $(L+1)$-by-1 matrix, then $\operatorname{kron}\left(\mathbf{I}_{N \times N}, \mathbf{B}\right)$ is an $N \times(L+1)$-by- $N \times 1$ matrix formed by taking all possible products between the elements of $\mathbf{I}$ and the matrix B.

\section{Proposed cICA-Based Detector}

The detection problem of maritime targets can be formulated as the following composite binary hypothesis test:

$$
\left\{\begin{array}{l}
H_{0}:\left\{\begin{array}{l}
x_{R 1}(t)=C_{\mathrm{CUT}}(t), \quad t=1, \ldots, T, \\
x_{R 2}(t)=C_{\mathrm{RCs}}(t), \quad \mathrm{RCs}=1, \ldots, P,
\end{array}\right. \\
H_{1}: \begin{cases}x_{R 1}(t)=\alpha T g_{\mathrm{CUT}}(t)+C_{\mathrm{CUT}}(t), & t=1, \ldots, T, \\
x_{R 2}(t)=C_{\mathrm{RCs}}(t), & \mathrm{RCs}=1, \ldots, P,\end{cases}
\end{array}\right.
$$

where $P$ is the number of available RCs. The null hypothesis $H_{0}$ occurs when the target is absent at the cell under test (CUT). The alternative hypothesis $H_{1}$ occurs when the target is present at the CUT. $x_{R 1}(t)$ is the received radar signal vector at the CUT, while $x_{R 2}(t)$ and $C_{\mathrm{RCs}}(t)$ are received at RCs. $T g_{\text {CUT }}(t)$ and $C_{\text {CUT }}(t)$ are the target and clutter return vectors at the CUT. $a$ is a coefficient adjusting SCR of the target. It corresponds to the mixing system coefficients of equation (1) such that, for example, in $x_{R 1}(t)$, we have $\alpha=\left(w_{12} / w_{11}\right)$.

There are three challenging problems in designing cICAbased detectors for monostatic radars: (1) The radar has a single receiving antenna, resulting in a single mixture, but we need at least two mixtures. (2) the cICA algorithm has multiple outputs, but we need to select an output at the end of the detector. (3) In many cICA algorithms, depending on the radar scenarios, the required sample size is too long to achieve proper separation performance. In this case, we propose three procedures in the blocks of the proposed detector of Figure 1 to solve these issues. This figure shows the proposed cICA-based detector for detecting the target in a coherent maritime fixed-site monostatic pulsed radar. It scans a patch of sea and stores the returned digitized complex-valued signal from a resolution cell in a matrix. We propose a method for choosing the second mixture when CUT is selected as the first mixture in the reference signal selection block and maritime data selection block. In these blocks, we assume that one range cell containing only clutter, an RC, can be used as the second mixture. Our experiments demonstrate the validity of this assumption in practical situations. In the following, to justify the method, we write the radar receiving signal from the CUT and a typical RC as follows:

$$
\begin{aligned}
x_{R 1}(t) & =w_{11} C(t)+w_{12} \operatorname{Tg}(t), \\
x_{R 2}(t) & =w_{21} C(t)+w_{22} \operatorname{Tg}(t)=w_{21} C(t), \\
w_{22} & =0, \quad \text { for a RC, }
\end{aligned}
$$

or in the convolutive cICA formulation,

$$
\begin{aligned}
& x_{R 1}(t)=\sum_{l=-\infty}^{+\infty} w_{11 l} C(t-l)+\sum_{l=-\infty}^{+\infty} w_{12 l} T g(t-l), \\
& x_{R 2}(t)=\sum_{l=-\infty}^{+\infty} w_{21 l} C(t-l)+\sum_{l=-\infty}^{+\infty} w_{22 l} T g(t-l) .
\end{aligned}
$$

In practical situations, we assume that $0 \leq l \leq L-1$. We can also assume that the target coefficients $w_{22 l}$ are zero in the second mixture $x_{R 2}(t)$. Thus, an RC can be used as one of the required mixtures. In conclusion, when the two mixtures $x_{R 1}(t)$ and $x_{R 2}(t)$ are processed by one of the cICA algorithms, it possibly results in two sources, i.e., $C(t)$ and $T g(t)$ if the target is present, as shown in our in our experiments. In fact, the proposed detector suppresses clutter using the clutter itself. Figure 2(a) displays the reference signal selection block. It computes the powers of all RCs, averages them, and then chooses an RC with the lowest power. It also computes the shape parameter of the K-distribution (see equations (28)-(30)) for three signals: the selected RC, the average signal, and the outputted maritime clutter. Finally, the block chooses the signal with the lowest shape parameter among these three signals as the reference signal.

The maritime data selection block of Figure 2(b) makes each detection decision in a coherent processing interval (CPI) on the CUT samples. $T_{\mathrm{CPI}}$ is the number of discrete 
TABLE 1: Measuring function [1].

\begin{tabular}{|c|c|c|c|c|}
\hline & $g^{[I]}(x)$ & $G^{[\mathrm{I}]}(x)$ & $g^{[\mathrm{II}]}(x)$ & $G^{[I I]}(x)$ \\
\hline 1 & $4 x^{3}$ & $x^{4}$ & $4 r^{3}$ & $r^{4}$ \\
\hline 2 & $\operatorname{sgn}(x) /(1+|x|)^{2}$ & $|x| /(1+|x|)$ & $1 /(1+r)^{2}$ & $r /(1+r)$ \\
\hline 3 & $|x|(20+|x|) /(10+|x|)^{2}$ & $x|x| /(10+|x|)$ & - & - \\
\hline 4 & $\left(1-x^{2}\right) /\left(1+x^{2}\right)^{2}$ & $x /\left(1+x^{2}\right)$ & - & - \\
\hline
\end{tabular}

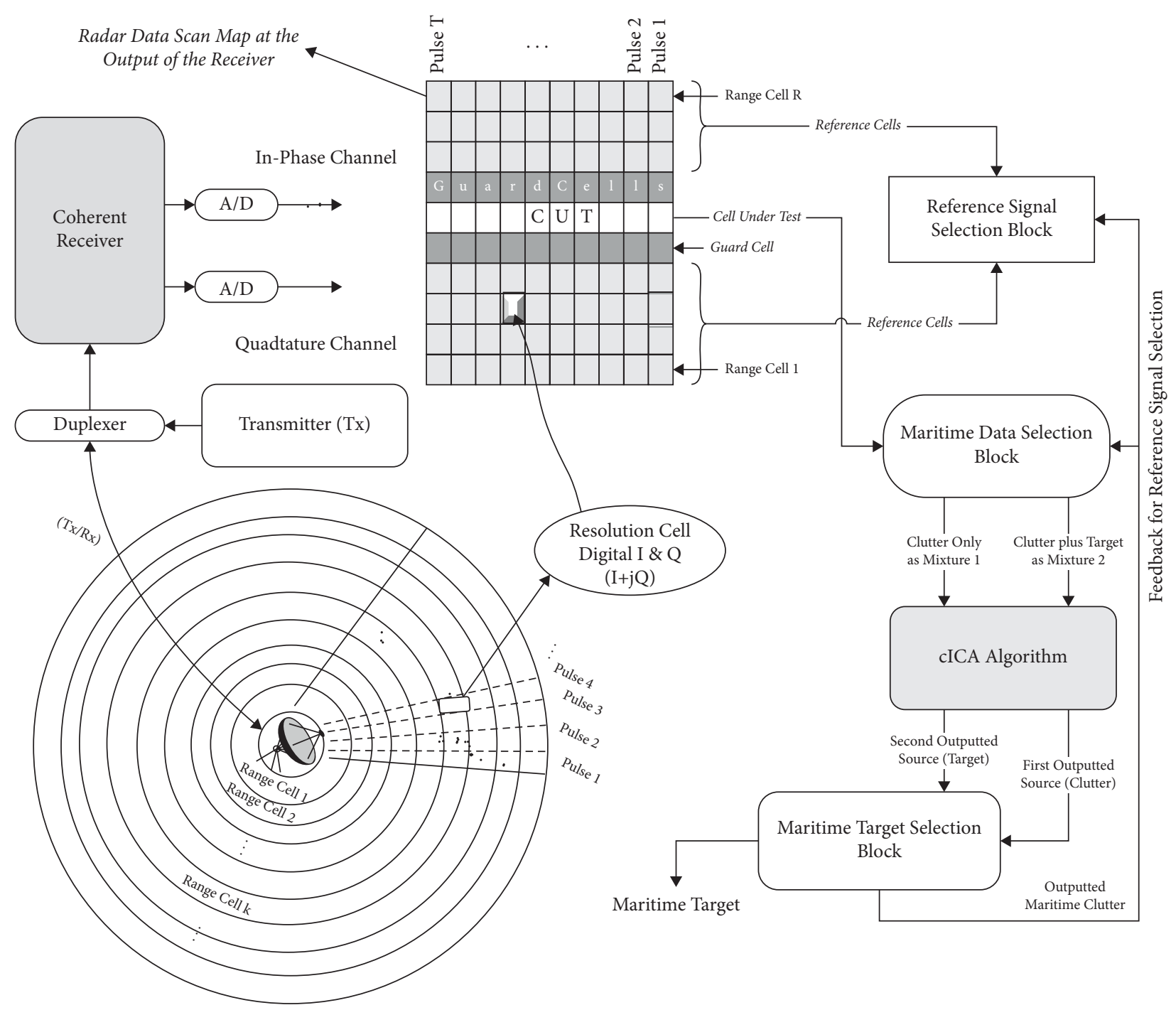

Figure 1: Proposed cICA-based detector.

time samples (sample size) of the CPI. The block catches two signals: the reference signal $x_{R 2}(t)$ and the CUT $x_{R 1}(t)$, both for $1<t<T$. It saves 512 samples of the two signals in two shift registers. Each shift register sends a signal of appropriate length to the cICA algorithm block, including new CPI samples. From now, on each detection decision, the shift register will fetch 8 new samples $\left(T_{\mathrm{CPI}}=8\right)$ and sends them to the cICA block along with 504 previously saved samples $\left(T_{\mathrm{Ref}}=504\right)$. This is repeated at each CPI. This means that the detection decision time is 8 , and the length of the processed signal is 512 . Note that $T_{\mathrm{CPI}}=T-T_{\mathrm{Ref}}$, and $T_{\mathrm{Ref}}$ is the sample size of the previously saved signal used to improve the separation performance, which can also be selected arbitrarily. Since the previously stored samples are available in any situation, it is feasible to use this method in practical situations. Therefore, the separation stage can be performed on any arbitrary sample size with any desired CPI for detection decision and integration.

Among the ICA algorithms, only CCEBM, CERBM, and IQ-STFICA can perform the separation task properly in the cICA block. This is due to the convolutive nature of real-life radar signals and their complicated and varying 


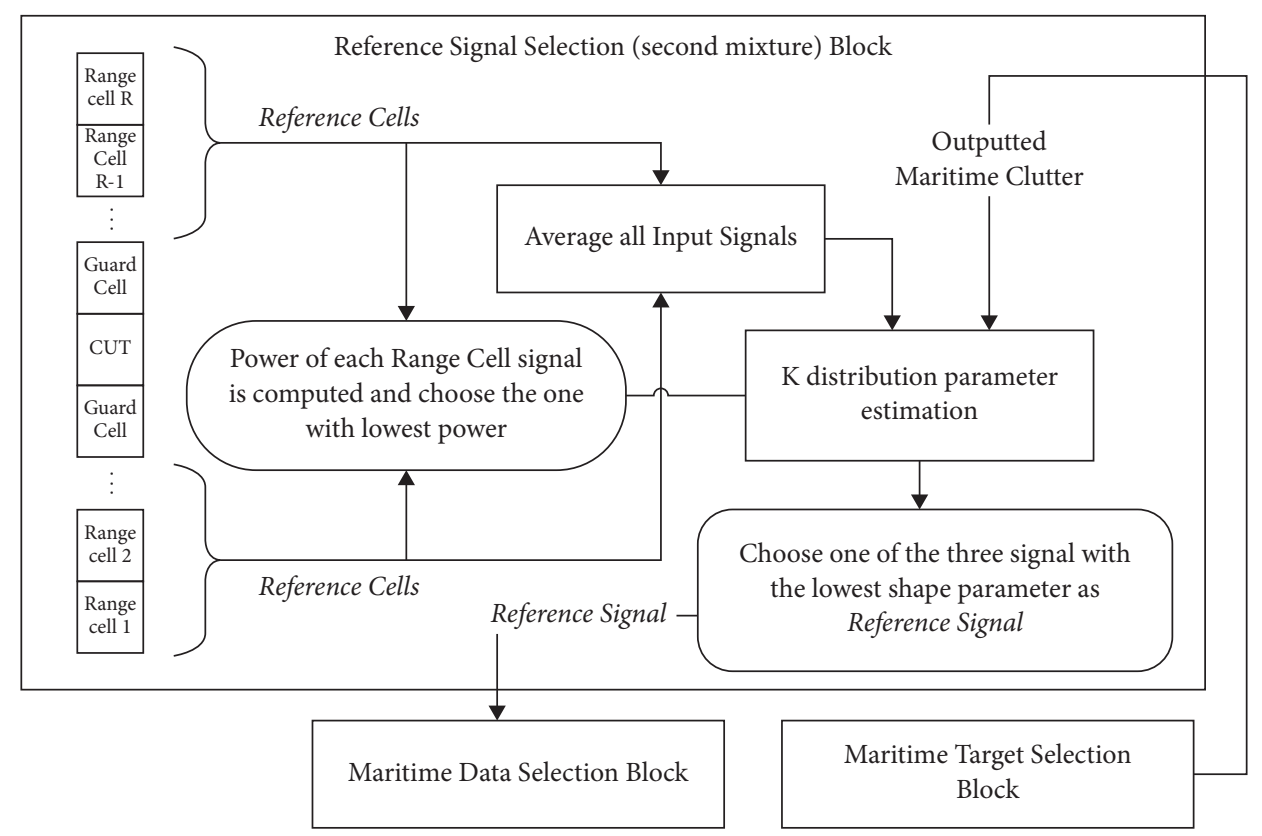

(a)

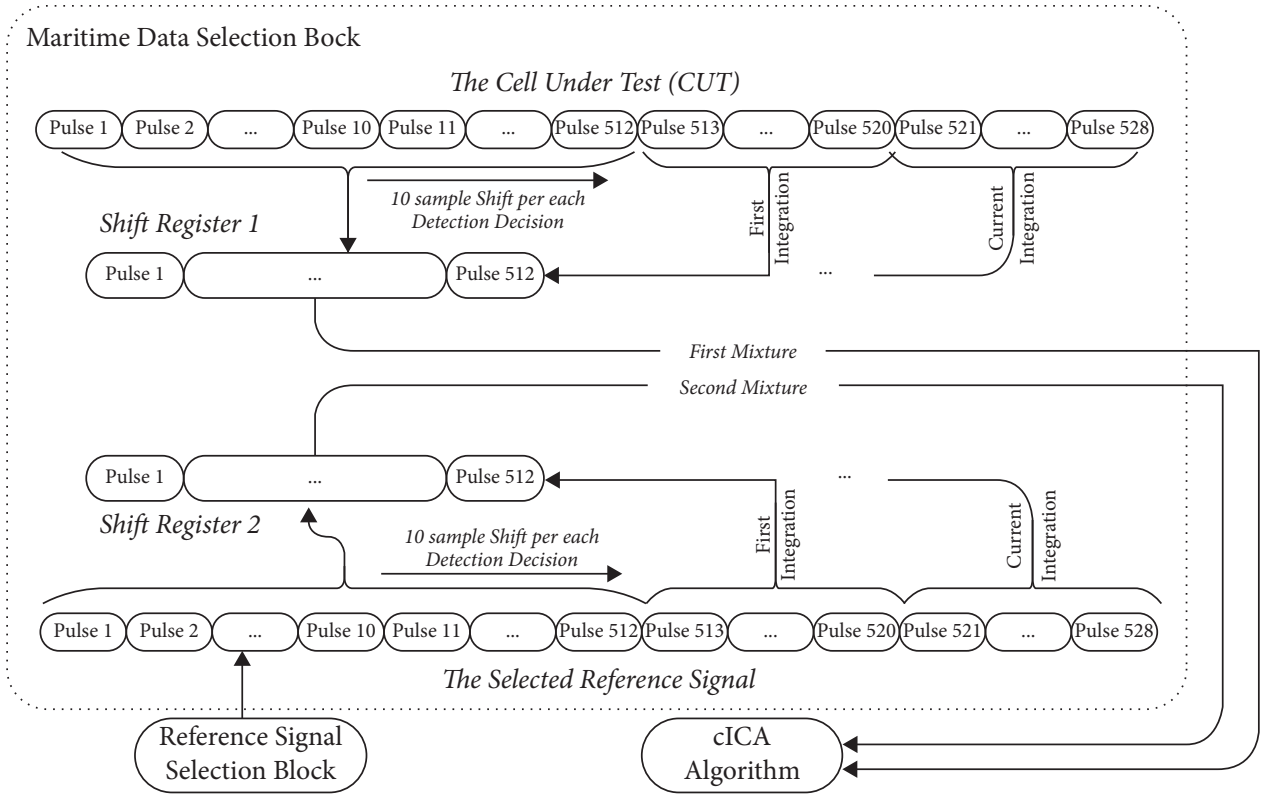

(b)

Figure 2: (a) Reference signal selection block. (b) Maritime data selection block.

characteristics. Although the CERBM is not a convolutive cICA algorithm, it performs the separation task very well on real-life IPIX radar data because it uses three types of diversity.

The maritime target selection block selects the target (if present) between the two outputted sources of the cICA algorithm. Since an output of the cICA algorithm is definitely clutter and the clutter statistical characteristic of the high-resolution sea is better suited to the K-distribution, we recommend the use of one $\mathrm{K}$-distribution parameter as a threshold to distinguish the clutter leading to the selected target. Many statistical distribution models, including Weibull, K, and compound-Gaussian, are proposed for sea clutter modeling [23], any of which can be adopted to achieve a threshold for target selection. Here, we use the most famous one, i.e., K-distribution with the PDF: 


$$
p(|C(t)|)=\frac{\sqrt{4(v / b)}}{2^{v-1} \Gamma(v)}\left(\sqrt{4\left(\frac{v}{b}\right)}|C(t)|\right)^{v} K_{v-1}\left(\sqrt{4\left(\frac{v}{b}\right)}|C(t)|\right), \quad 0 \leq|C(t)|<\infty,
$$

where $\Gamma(\cdot)$ is the gamma function, $K_{v-1}(\cdot)$ represents the modified Bessel function of the third kind of order of $v-1$, and $v$ denotes the shape parameter. Moreover, $b$ is known as the scale parameter. The parameters of the K-distribution PDF can be estimated using the classical method of moments (MoM), calculating the first and second empirical and theoretical moments [33], using these two equations:

$$
\begin{aligned}
b & =E\left\{|C(t)|^{2}\right\}, \\
\frac{4 v}{\pi}\left(\frac{\Gamma(v)}{\Gamma(v+(1 / 2))}\right) & =\frac{E\left\{|C(t)|^{2}\right\}}{(E\{|C(t)|\})^{2}},
\end{aligned}
$$

where the $k$ th-order moment is replaced by its sample estimate:

$$
\widehat{E}\left\{|C(t)|^{k}\right\}=\frac{1}{T_{\mathrm{CPI}}} \sum_{t=1}^{T_{\mathrm{CPI}}}|C(t)|^{k} \longrightarrow E\left\{|C(t)|^{k}\right\} .
$$

In the marine target selection block, the shape and scale parameters are first estimated using equations (29) and (30), and these parameters are then compared with precalculated thresholds. The target selection procedure in the maritime target selection block is illustrated in Figure 3(a) and is based only on shape parameters.

Figure 3(b) depicts the flowchart of the proposed detector. It is an open structure because we can utilize any cICA algorithm for detection. To calculate the threshold in the training branch, when the CUT contains only clutter (RCs), we used 100,000 time-samples to estimate the shape (scale) parameters of the cICA block output source. A total of $100,000(100 / 0.001)$ is the number of Monte Carlo tests (100/PFA) required to achieve the desired probability of false alarm (PFA). Considering the samples available in the RCs of the IPIX datasets, it is set to 0.001 in this paper. Since the elements of the estimated shape (scale) parameter vector are arranged in ascending order, if we choose a shape parameter that is the $[((100 / \mathrm{PFA})-100)]$ th (99900th) element of this shape parameters vector, we obtain the threshold, i.e., a_output_ref (or b_output_ref). To compute the detection probability (PD) at each SCR level, 1000 Monte Carlo tests to the CUT are performed in the detection branch of Figure 3(b). To compute the detection probabilities for reallife targets, due to the limited number of available samples at the CUT of each dataset of the IPIX radar, 100 Monte Carlo tests are performed. Finally, the entire detection process of the proposed cICA-based detector is presented in the detection branch of Figure 3(b).

\section{Performance Analysis}

In the performance evaluation of the cICA-based detector, we conducted extensive experiments on real-life data and synthetic simulated targets. The specifications of the
McMaster IPIX radar and the datasets are presented in Tables 2 and 3, respectively. To evaluate the detector's experimental ability, we employed 10 datasets from the Dartmouth database of IPIX radar and 10 datasets from Grimsby. For each dataset, the SCR for the real-life target is estimated using this formula [33]:

(i) PRF is $1000 \mathrm{~Hz}$ with the pulse width of $200 \mathrm{~ns}$ in a static scan mode (stare or dwell mode) with a pencil beam of 0.9 degree.

(ii) Datasets 1-10 are from Dartmouth database recorded at range resolution $30 \mathrm{~m}$, sampled at $15 \mathrm{~m}$, and has 14 range cells with 217 time samples. Datasets 11-20 are from Grimsby database recorded at range resolution $30 \mathrm{~m}$, sampled at $15 \mathrm{~m}$, and has 28 range cells with $6 \times 10^{4}$ time samples.

(iii) The range resolution of the datasets 18 th and 19th is $15 \mathrm{~m}$ (pulse widths $100 \mathrm{~ns}$ ) and $9 \mathrm{~m}$ (pulse widths $60 \mathrm{~ns})$, respectively.

(iv) In Dartmouth datasets, the object observed by the radar is a $1 \mathrm{~m}$ diameter styrofoam hall which is wrapped in radar reflective material and which floats on the ocean surface on an anchor line at about $2.5 \mathrm{~km}$ offshore. For the Grimsby datasets, the target is a small floating boat dragged by an anchor leading to very little backward and forward movement caused by swells.

$$
\mathrm{SCR}=\frac{P_{\text {total }}-P_{\text {clutter }}}{P_{\text {clutter }}}
$$

$P_{\text {clutter }}$ and $P_{\text {total }}$ are the clutter and clutter plus target power, respectively, calculated by averaging the power of RCs. Note that in all the experiments, the filter length parameter $L$ for the CCEBM algorithm is set to 3. Moreover, this parameter is set to 10 for the STFICA algorithm like what is specified in [29]. Compared with IQ-STFICA, the filter length used in CCEBM is different due to the different separation stages in the two algorithms. For the CERBM algorithm, the filter length $p$ is set to 1 based on the lengths suggested in [30] and what we actually observed in the experiments. Also, we do not use the lite version of the CERBM. We understood through the experiments that the filters length of the algorithms suggested in $[29,30]$ is optimal for radar detection applications. Although the prewhitening stages of CCEBM an IQ-STFICA are the same, but due to their differences in the separation stage, CCEBM performs properly with filter length $3 \leq L \leq 10$. Therefore, we use 3 as the optimal filter length to reduce computational time in CCEBM. 


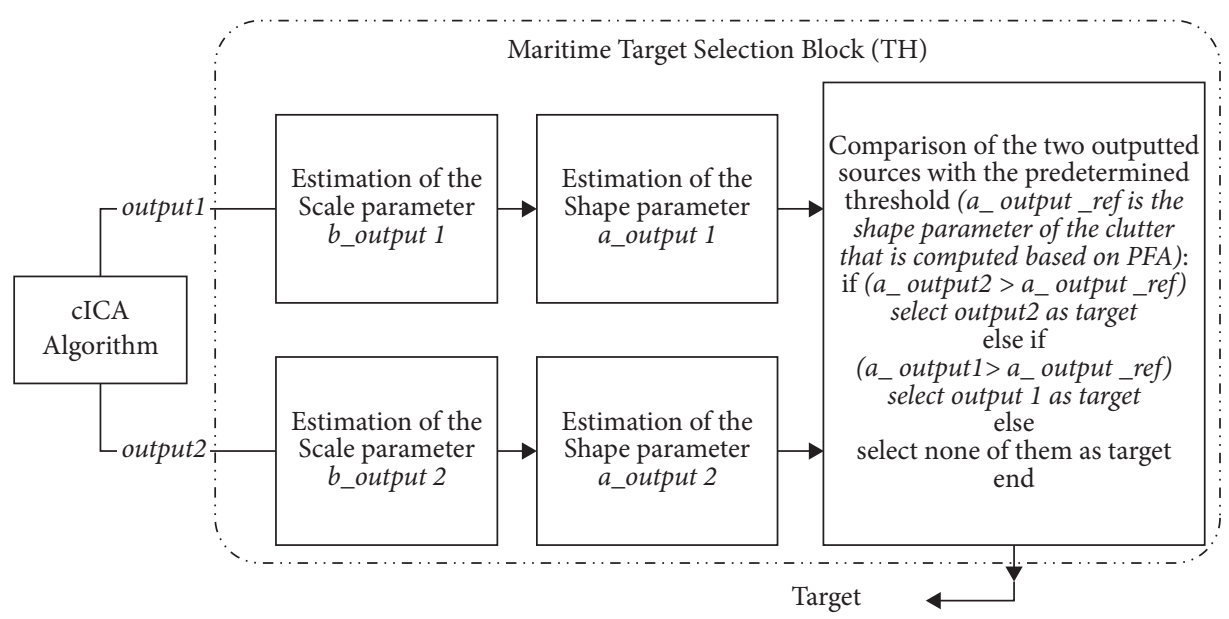

(a)

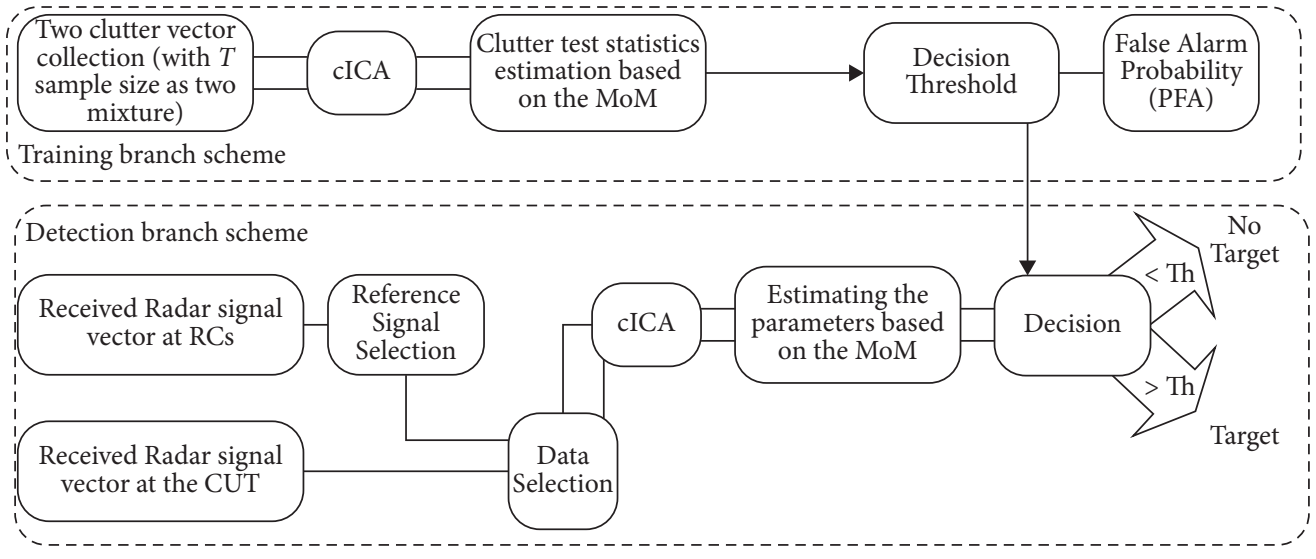

(b)

Figure 3: (a) Maritime target selection block. (b) Flowchart of the proposed cICA-based detector.

The maximum number of iterations is set to 50 although the CCEBM and STFICA often converge in less than 10 iterations.

The synthetic targets simulated and used in this section follow the Swerling models. A synthetic lognormal target with a radar cross section (RCS) following lognormal distribution is also utilized. The specifications of the synthetic targets are summarized in Table 4 . For the synthetic targets, we have

$$
\alpha T g(t)=\alpha \check{\mathrm{R}} \exp \left[\mathrm{j}\left(2 \pi \mathrm{tf}_{\mathrm{d}} \mathrm{T}_{\mathrm{r}}+\varphi\right)\right],
$$

where $\check{\mathrm{R}}$ is a random variable denoting the RCS, $f_{d}$ is the Doppler frequency shift, $T_{r}$ is the pulse repetition interval, $\varphi$ is a random variable that is uniformly distributed in the interval $0 \leq \varphi \leq 2 \pi$, and $\alpha$ is a deterministic constant to control the SCR and computed by $\mathrm{SCR}=10 \log _{10}\left(\alpha / P_{\text {Clutter }}\right)$ (in dB).

4.1. Experiments for Detection of the Synthetic Targets Injected into the Real-Life IPIX Radar Sea Clutter. Herein, the results of experiments for the detection of synthetic targets injected in real-life sea clutter are presented qualitatively and quantitatively. The TD image of the range cell 1 of file \#17 (that contains only sea clutter) is shown in Figure 4(a) with $T=20000$. We inject Tg-SW34 (Figure 4(b)) into it with $\mathrm{SCR}=-5 \mathrm{~dB}$. The TD image of this mixture is depicted in Figure 4(c). Then, this mixture is passed through the detector as the CUT while CCEBM is utilized in the cICA algorithm block. The range cell 2 of file \#17 is used as the second mixture. The TD images of the output sources from the CCEBM algorithm are displayed in Figures 4(d) and 4(e). The result is obtained, and one of the sources extracted is the target. The other one is a signal with a TD similar to file \# 17. Note that if the cICA detector uses CERBM or IQ-STFICA in the cICA block instead of our CCEBM algorithm, it leads to similar results qualitatively. The results are unacceptable for other algorithms, e.g., CEBM, nc-FastICA, JADE, and even native PCA or LDA.

The power curves are depicted in Figures 4(f)-4(j). Here, the synthetic targets are injected into the RCs of files \#17 and \#26 one by one (except for the range cell 1 as the reference signal). The cICA algorithm block obtains the mixtures with $T=512$ and uses CCEBM, CERBM, and IQ-STFICA algorithms, respectively. Evidently, the proposed CCEBM algorithms can accurately extract targets with low SCRs. In all cases, the proposed detector can correctly detect targets with $\mathrm{PD}=1$ in SCRs greater than -10 regardless of whether 
TABle 2: Specifications of the McMaster IPIX radar datasets.

\begin{tabular}{lcc}
\hline Specifications & Dartmouth & Grimsby \\
\hline Radar scan mode & & Static \\
Pulse repetition frequency & & $1000 \mathrm{~Hz}$ \\
Radar beam width & $30 \mathrm{~m}$ & $0.9^{\circ}$ \\
Range resolution & $2574-2769 \mathrm{~m}$ & $15 \mathrm{~m}$ \\
Range & 131072 & - \\
Number of pulses & 14 & 60000 \\
Number of cells & 28 \\
RF frequency & H or V polarization, switchable pulse-to-pulse \\
Transmitter & $20-200$ ns \\
Polarization & Fully coherent reception \\
Pulse width & $2.39 \mathrm{GHz}$ diameter parabolic dish \\
Receiver & & 2 peak power TWT \\
Antenna & & \\
\hline
\end{tabular}

TABLE 3: The used McMaster IPIX radar datasets.

\begin{tabular}{|c|c|c|c|c|c|c|c|c|c|}
\hline & \multirow{2}{*}{ File number } & \multirow{2}{*}{ Data label } & \multirow{2}{*}{ Guard cells } & \multirow{2}{*}{ CUT } & \multirow{2}{*}{ Wave height } & \multicolumn{4}{|c|}{ SCR $(\mathrm{dB})$ of the target } \\
\hline & & & & & & $\mathrm{HH}$ & $\mathrm{HV}$ & $\mathrm{VH}$ & $\mathrm{HH}$ \\
\hline 1 & $\# 54$ & 19931111_163625_starea & $7,9,10$ & 8 & 0.7 & 18.438 & 18.563 & 18.563 & 9.141 \\
\hline 2 & $\# 30$ & 19931109_191449_starea & 6,8 & 7 & 0.9 & -0.469 & 3.672 & 3.672 & 2.000 \\
\hline 3 & $\# 31$ & 19931109_202217_starea & $6,8,9$ & 7 & 0.9 & 6.641 & 7.578 & 7.578 & 8.359 \\
\hline 4 & $\# 310$ & 19931118_162155_stare & $6,8,9$ & 7 & 0.9 & 2.344 & 5.000 & 5.000 & -1.563 \\
\hline 5 & \#311 & 19931118_162658_stare & $6,8,9$ & 7 & 0.9 & 12.266 & 15.000 & 15.000 & 8.906 \\
\hline 6 & $\# 320$ & 19931118_174259_stare & $6,8,9$ & 7 & 0.9 & 12.109 & 14.000 & 14.000 & 6.953 \\
\hline 7 & \#40 & 19931110_001635_starea & $5,6,8$ & 7 & 0.9 & 9.766 & 13.200 & 13.200 & 11.250 \\
\hline 8 & $\# 26$ & 19931108_220902_starea & 6,8 & 7 & 1.0 & 4.297 & 6.000 & 6.000 & 5.859 \\
\hline 9 & $\# 280$ & 19931118_023604_stare & 7,10 & 8 & 1.4 & 4.063 & 7.500 & 7.500 & 4.531 \\
\hline 10 & \#17 & 19931107_135603_starea & $8,10,11$ & 9 & 2.1 & 18.750 & 13.280 & 13.350 & 3.750 \\
\hline 11 & $\# 21 \mathrm{G}$ & 19980204_202225_ANTSTEP & $23,25,26$ & 24 & - & 5.000 & 27.344 & 29.297 & 4.453 \\
\hline 12 & $\# 22 \mathrm{G}$ & 19980204_202525_ANTSTEP & $6,8,9$ & 7 & - & 4.922 & 25.000 & 26.953 & 4.375 \\
\hline 13 & \#6G & 19980204_163113_ANTSTEP & $23,25,26$ & 24 & - & -2.266 & 17.344 & 17.188 & -2.188 \\
\hline 14 & \#41G & 19980205_171437_ANTSTEP & $6,8,9$ & 7 & - & 0.938 & 20.547 & 20.938 & 0.313 \\
\hline 15 & \#46G & 19980205_180558_ANTSTEP & $6,8,9$ & 7 & - & 8.203 & 23.900 & 23.125 & 7.891 \\
\hline 16 & $\# 65 \mathrm{G}$ & 19980212_195704_ANTSTEP & $6,8,9$ & 7 & - & 10.000 & 25.781 & 24.375 & 9.374 \\
\hline 17 & $\# 82 \mathrm{G}$ & 19980223_164055_ANTSTEP & $30,32,33$ & 31 & - & -1.875 & 20.781 & 20.650 & -1.328 \\
\hline 18 & \#91G & 19980223_173317_ANTSTEP & $31,33,34$ & 32 & - & 1.484 & 13.906 & 18.125 & 3.672 \\
\hline 19 & $\# 92 \mathrm{G}$ & 19980223_173950_ANTSTEP & $28,30-34$ & 29 & - & 2.188 & 13.203 & 13.359 & 1.485 \\
\hline 20 & $\# 204 \mathrm{G}$ & 19980304_184537_ANTSTEP & 20,22 & 21 & - & 8.000 & 7.109 & 15.781 & 8.750 \\
\hline
\end{tabular}

the target is in the noise Doppler unit. The probabilities of detection of Tg-SW0 versus SCR for $T=512,1024$, and 2048 are shown in Figure 4(k), while the CCEBM is utilized in the cICA block. We can see that the proposed detector with the proposed CCEBM or CERBM and IQ-STFICA has excellent detection performance in a wide variety of situations, whether the sea state is high or not.

Figure 4(1) compares the cICA-based detector's performance when it employs CCEBM, CERBM, IQ-STFICA, and CFPA with $T=128, T_{\text {Ref }}=0$, and $T_{\mathrm{CPI}}=128$, for embedded Swerling II target (the Tg-SW2) in file \#26. This figure also illustrates the performance of CA-CFAR for this target. CCEBM has the best performance in most cases, and CERBM shows a stable performance in the medium, unlike IQ-STFICA, which performs very poorly in some cases, especially when the sample size is small. We compare the detection performance with CA-CFAR to demonstrate the superiority of the detector over a conventional detector.
Since the Tg-SW2 is a moving target, this experiment also indicates that the detector can detect moving Swerling targets with very low SCR as well as stationary ones. It can be utilized without need for Doppler processing techniques.

4.2. Performance Comparison of the Proposed Detector for Detection of Real-Life Sea-Surface Floating Small Targets Existed in Real-Life IPIX Datasets. For a full comparison, the proposed detector is employed to detect real-life sea-surface small floating targets existing in IPIX datasets. Many novel detection methods have been proposed to solve this type of problem $[12,14,15,19]$. Figure 5 illustrates the TD images of the detected target from primary cells of five datasets using the cICA-based detector with CCEBM. It can be seen visually that the proposed detector successfully detects the target with the highest accuracy. The Doppler offset of the targets indicates a quasiperiodic trend in TD images. Each 
TABLE 4: Specifications of the synthetic Swerling and lognormal targets.

\begin{tabular}{|c|c|c|c|c|c|c|}
\hline Specification & Swerling 0 & Swerling I & Swerling II & Swerling III and IV & $\begin{array}{l}\text { Lognormal } \\
\text { target }\end{array}$ & $\begin{array}{c}\text { Real IPIX } \\
\text { target }\end{array}$ \\
\hline Used name & Tg-SW0 & Tg-SW1 & Tg-SW2 & Tg-SW34 & Tg-ext & Tg-real \\
\hline Target RCS & Constant & $\begin{array}{l}\text { Chi- } \\
\text { square }\end{array}$ & $\begin{array}{l}\text { Chi- } \\
\text { square }\end{array}$ & Chi-square & Lognormal & - \\
\hline Degrees of freedom & - & 2 & 2 & 4 & & \\
\hline Number of objects & 1 & 1 & 1 & 3 & 1 & 1 \\
\hline $\begin{array}{l}\text { Intimate velocity of objects } \\
(\mathrm{m} / \mathrm{s})\end{array}$ & $0.35 \mathrm{~m} / \mathrm{s}$ & $0.35 \mathrm{~m} / \mathrm{s}$ & $2 \mathrm{~m} / \mathrm{s}$ & First: 1 , second: 0.2 , third: -2 & 0.5 & 0 \\
\hline Velocity & $0.35 \mathrm{~m} / \mathrm{s}$ & $0.35 \mathrm{~m} / \mathrm{s}$ & Variant & $\begin{array}{c}\text { First: variant, second: } 0.2 \text {, third: } \\
\text { variant }\end{array}$ & Variant & Near zero \\
\hline Acceleration & 0 & 0 & $1 \mathrm{~m} / \mathrm{s}^{2}$ & First: 1, second: 0 , third: random & Variant & Near zero \\
\hline Doppler frequency & $21.91 \mathrm{~Hz}$ & $21.91 \mathrm{~Hz}$ & Variant & $\begin{array}{c}\text { First: variant, second: } 12.52 \mathrm{~Hz} \text {, third: } \\
\text { variant }\end{array}$ & Variant & Near zero \\
\hline Maneuvering & No & No & Yes & Object 1 and 2: no, object 3: yes & Yes & No \\
\hline
\end{tabular}

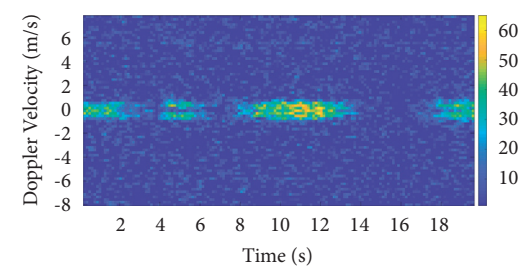

(a)

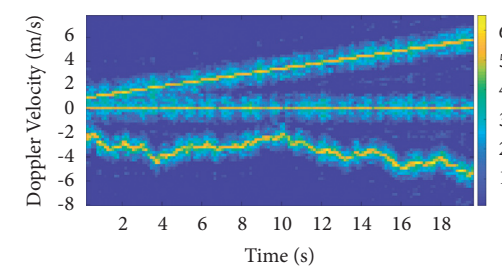

(d)

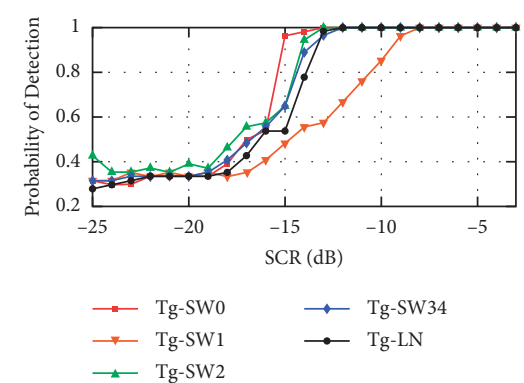

(g)

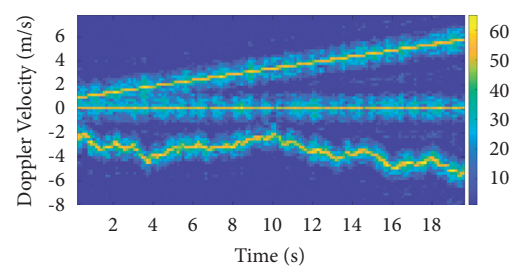

(b)

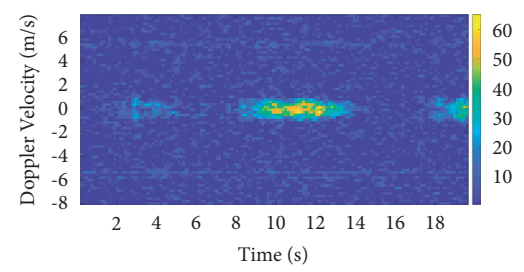

(e)
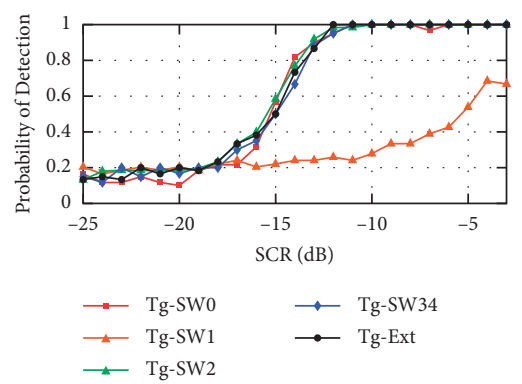

(h)

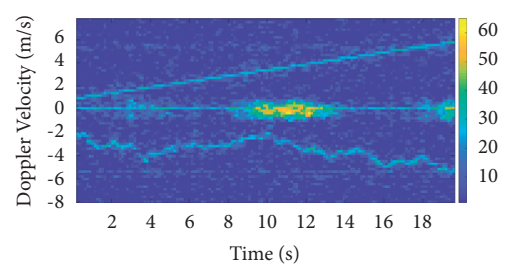

(c)
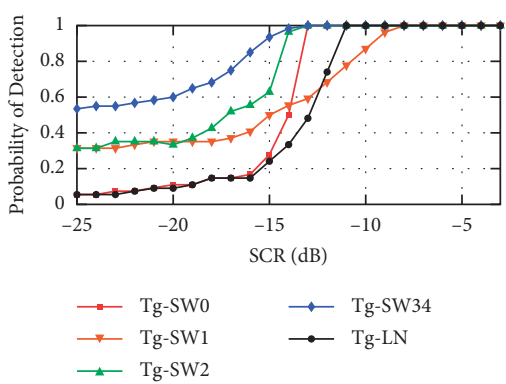

(f)

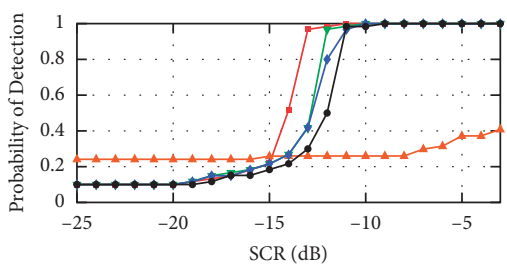

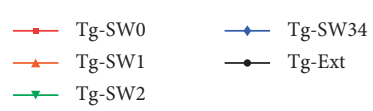

(i)

Figure 4: Continued. 


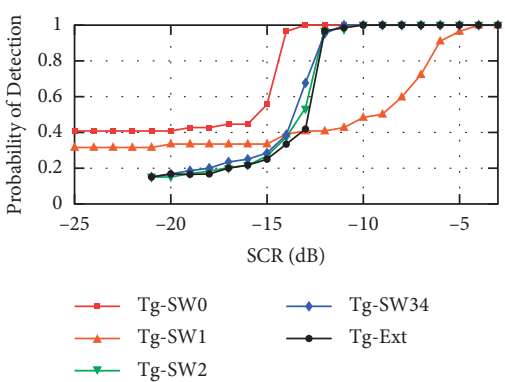

(j)

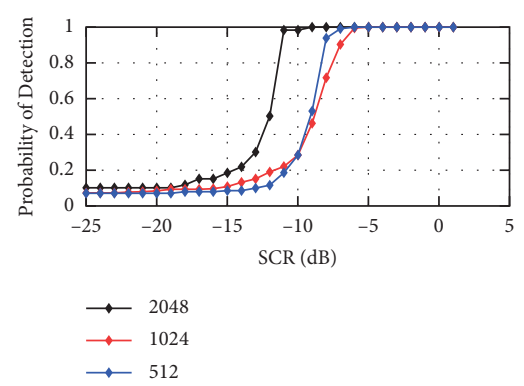

$(\mathrm{k})$

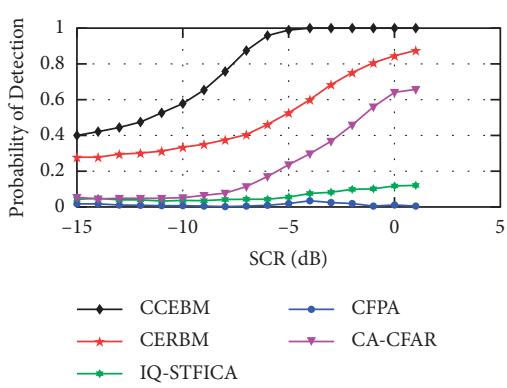

(1)

Figure 4: Testing the cICA-based detector in low and high sea states, i.e., the datasets 8 and 10 (file \#26 and \#17). The time-Doppler image of (a) the first $T=20000$ (20 seconds) of the range cell 1 of file \#17; (b) the simultaneous Swerling III and IV synthetic target, i.e., Tg-SW34, with $T=20000$; (c) the Tg-SW34 injected into the first 20 seconds of the range cell 1 with SCR $=-5 \mathrm{~dB}$; (d) the outputted target from the mixture of Figure 4(c) utilizing the CCEBM algorithm; (e) the outputted clutter from the mixture of Figure 4(c) utilizing the CCEBM algorithm. PD versus SCR (power curve) with PFA sets to be 0.001 , for all synthetic targets, when the proposed detector utilizes: (f) CCEBM, file \#17 with $T=512, T_{\text {Ref }}=0$, and $T_{\mathrm{CPI}}=512$; (g) CERBM, file \#17 with $T=512, T_{\text {Ref }}=0$, and $T_{\mathrm{CPI}}=512$; (h) IQ-STFICA, file \#17 with $T=4608, T_{\text {Ref }}=4096$, and $T_{\mathrm{CPI}}=512$; (i) CCEBM, file \#26 with $T=512, T_{\text {Ref }}=0$, and $T_{\mathrm{CPI}}=512$; (j) CERBM, file \#26 with $T=512, T_{\text {Ref }}=0$, and $T_{\mathrm{CPI}}=512$; (k) CCEBM, for detection of the target Tg-SW0 in file \#26 with $T=512, T_{\text {Ref }}=0$, and $T_{\text {CPI }}=512,1024,2048$. (l) Comparison of the cICA-based detector performance when it uses CCEBM, CERBM, IQSTFICA, and CFPA, with $T=128, T_{\text {Ref }}=0$, and $T_{\text {CPI }}=128$, for embedded Swerling II target (the Tg-SW2) in \#file 26. This figure also shows CA-CFAR performance for this target.

floating small target is pushed and pulled by sea waves, more in the higher sea state, with a period relevant to the period of sea waves. Expectedly, the average velocity of sea waves is near zero.

Finally, the proposed detector's performance is compared with several novel detectors. In Table 5, the detection probability for the primary cells of the 10th and 8th datasets for four types of polarization is computed. The bold font in each column is the highest detection probability. The proposed detector with CCEBM always has the best detection performance. In addition, detection probabilities for all 20 datasets of Table 3 are given in Figure 6(a) compared with the feature-based detector using three TF features [14], the trifeature-based detector [13], and the fractal-based detector [10,11]. Besides, Figure 6(b) shows the performance of the detector against two recently proposed and effective detectors, i.e., feature-compressionbased [19] and adaptive composite GLRT detectors [35]. In terms of detection performance, the proposed detector outperforms in all 20 datasets in Figures 6(a) and 6(b). Finally, Figure 6(c) displays the receiver operation feature curves (ROCs) of the proposed detector, 3D polarimetricfeature detector [19], and trifeature-based detector [14] at $\mathrm{HH}, \mathrm{HV}, \mathrm{VH}$, and VV. The superior performance of the proposed detector is apparent.

The IPIX radar works at the X-band which will be affected by rain $[36,37]$. Rain changes the radar backscatter through volume scattering, weakening due to raindrops within the interceding air, and variations in ocean surface harshness due to rain hitting the sea surface. Since our detector uses the clutter itself to extract the target from the clutter, the type of clutter in the background is not important. This means that even if the clutter is contaminated by rain clutter, the detector can detect the target because it uses the contaminated clutter to suppress the clutter online.
4.3. Sample Size Dependency and Computational Time of the Proposed CCEBM and Other cICA Algorithms. The computational time of the proposed CCEBM algorithm against some other fast and well-known algorithms is presented in Figure $7(\mathrm{a})$. We utilize a PC laptop to compute these computational times in corresponding MATLAB code. The machine and coding environment of all the algorithms are the same. The computational time of CCEBM is shorter than the lite version of the CERBM. Furthermore, its computational time is only slightly more than CEBM which is one of the fastest cICA algorithms owing to its line search procedure.

In Figure 7(b), the probability of detection is depicted versus the sample size $T$ for SCR $=-5$. The detector's performance remains unchanged when $T$ is more than 512 . Therefore, we choose $T=512$ as the optimum sample size of the input signal to the cICA block. If a larger sample size is chosen, it may be better for the separating task in some cases, but it consumes the computational time and may reduce the detection performance in practical situations due to the radar signals' nonstationary nature. CCEBM requires minimum but IQ-STFICA requires maximum sample size. The performance of the detector with CCEBM is superior even with $T=128$. In conclusion, the proposed CCEBM is a fast convolutive cICA algorithm with an acceptable computational time, which can make the detection decision in an arbitrarily CPI.

4.4. Discussion. Based on the above experiments, several conclusions can be drawn. First, the detector can separate moving and stationary targets with very low SCRs and different RCS such as Swerling and lognormal models. In this case, its performance is better than the conventional CA-CFAR and several novel detectors. Second, CCEBM 


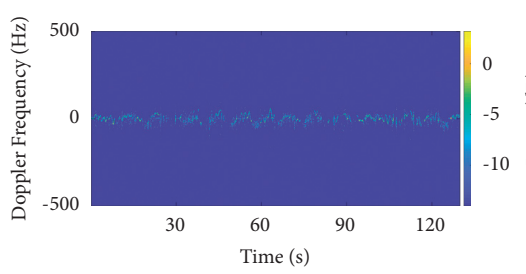

(a)

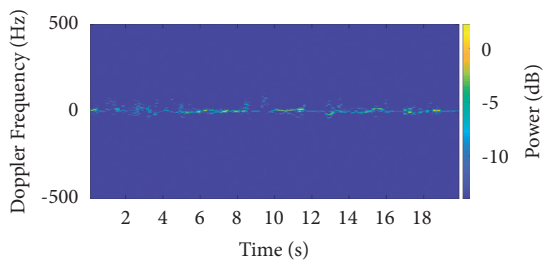

(d)

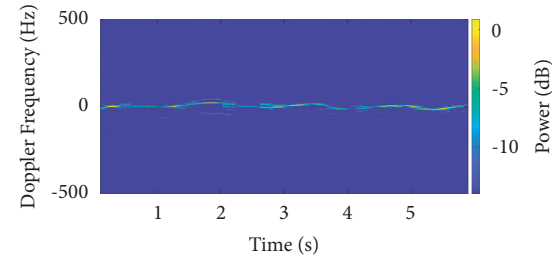

(b)

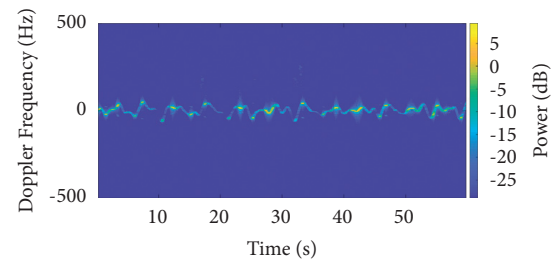

(e)

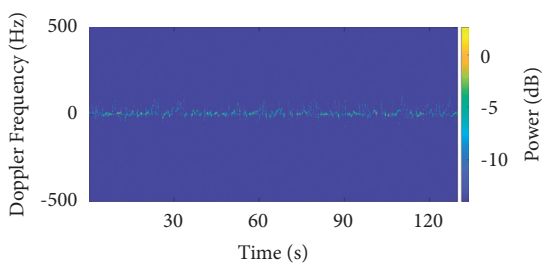

(c)

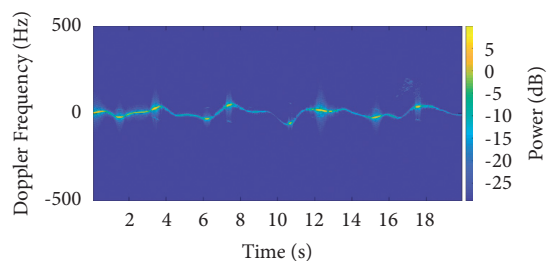

(f)

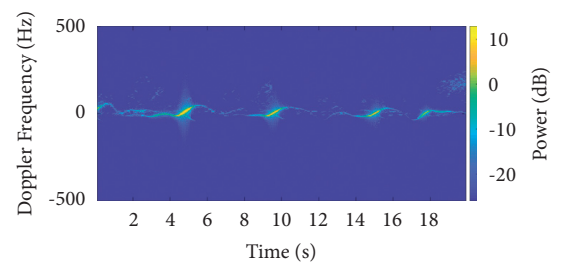

(g)

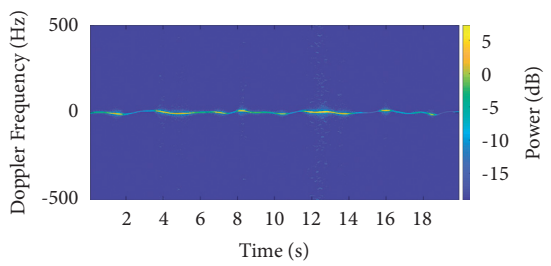

(h)

FIgURE 5: Extraction of real-life sea-surface small target from the IPIX datasets. The outputted target from (a) the range cell 9 of the dataset 10th (file \#17); (b) the first 6 seconds of the range cell 9 of the dataset 10th (file \#17); (c) the range cell 7 of the dataset 1 (file \#26); (d) the first 20 seconds of the range cell 7 of the dataset 1 (file \#26); (e) the range cell 7 of the dataset 3 (file \#22G); (f) the first 20 seconds of the range cell 7 of the dataset 3 (file \#22G); (g) the first 20 seconds of the range cell 29 of the dataset 4 (file \#92G); (h) the first 20 seconds of the range cell 9 of the file \#58 of Grimsby datasets, i.e., 19980206_195948_ANTSTEP.CDF, which have $3 \mathrm{~m}$ resolution (pulse width $20 \mathrm{ns).}$

TABle 5: Comparison of detection performance at the two datasets of the IPIX database.

\begin{tabular}{lcccccccc}
\hline cICA-detector parameters & \multicolumn{4}{c}{$T=1024, T_{\text {Ref }}=0$, and $T_{\text {CPI }}=1024$} \\
\hline Data label & \multicolumn{3}{c}{ Dataset 2 $(\# 17$ RC \#9) } & \multicolumn{4}{c}{ Dataset 1 (\#26 RC \#7) } \\
Polarization & VV & VH & HV & HH & VV & VH & HV & HH \\
RQA [15] & 0.014 & 0.127 & 0.132 & 0.382 & 0.311 & 0.332 & 0.338 & 0.328 \\
Joint-fractal-based [12] & 0.221 & 0.573 & 0.557 & 0.496 & 0.351 & 0.405 & 0.405 & 0.282 \\
Feature-based detector using three TF features [20] & 0.334 & 0.519 & 0.489 & 0.641 & 0.504 & 0.725 & 0.733 & 0.748 \\
Proposed detector with CCEBM & $\mathbf{0 . 5 3 5 4}$ & $\mathbf{0 . 6 2 2 3}$ & $\mathbf{0 . 6 1 0 4}$ & $\mathbf{0 . 6 6 1 4}$ & $\mathbf{0 . 5 4 1 5}$ & $\mathbf{0 . 8 1 3 4}$ & $\mathbf{0 . 8 2 8 7}$ & $\mathbf{0 . 8 4 0 9}$ \\
Proposed detector with CERBM & 0.3792 & 0.5298 & 0.5165 & 0.5409 & 0.5120 & 0.5177 & 0.5365 & 0.5488 \\
Proposed detector with IQ-STFICA & 0.0394 & 0.0079 & 0.0061 & 0.0157 & 0.0076 & 0.0052 & 0.0147 & 0.0179 \\
\hline
\end{tabular}

attains the highest detection performance when used in the cICA-based detector compared with CERBM and IQSTFICA, the latter having the worst performance. Other mentioned cICA algorithms cannot detect radar targets. Sometimes, simple PCA and LDA [38] are very powerful, but they fail to separate the real-life target from clutter for radar target detection. Third, in a complex problem of seasurface small floating target detection, the detector has the best performance compared with several novel detectors. Fourth, the detector can perform detection in arbitrary observation time. The detector's performance is superior even with 64 time samples, thereby extending its application to radar scenarios in the future. Fifth, the detection probabilities of different files in the IPIX datasets do not follow a certain pattern of clutter-to-noise ratio (CNR), SCR, or clutter shape parameter. In other words, the detection performance is not very sensitive to these parameters which vary with sea state changes. The detector has similar performance in higher and lower sea states. Figure 5 illustrates the temporal and the Doppler evolution obtained by processing the data from the range cells. The targets extracted from sea waves exhibit a common periodic trend with their own reciprocal delay. The Doppler varies below several Hertz. The effect of noise in the temporal and spectral features of the received radar returns may be quite different, depending on the CNR. According to the estimates, the CNR ranges from about $-5 \mathrm{~dB}$ when the texture is minimum up to about $80 \mathrm{~dB}$ when the texture is maximum. For spiky sea clutter, we generally have $v \in[0.1,2]$. Nevertheless, the proposed detector's detection performance is approximately the same in high and low sea states, suggesting that it is a robust detector for different RCSs, SCRs, CNRs, and shape parameters. 


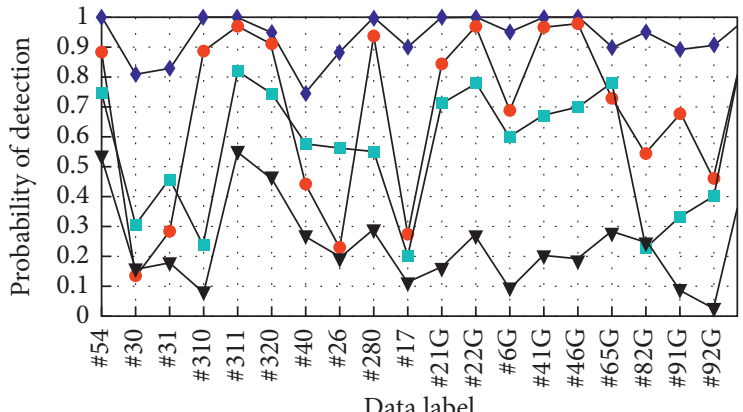

Data label

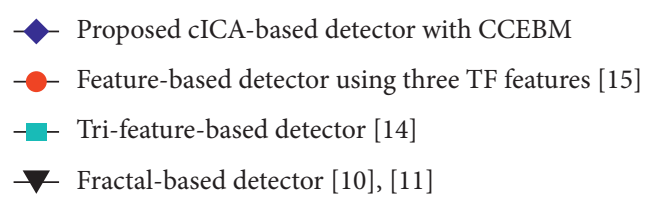

(a)

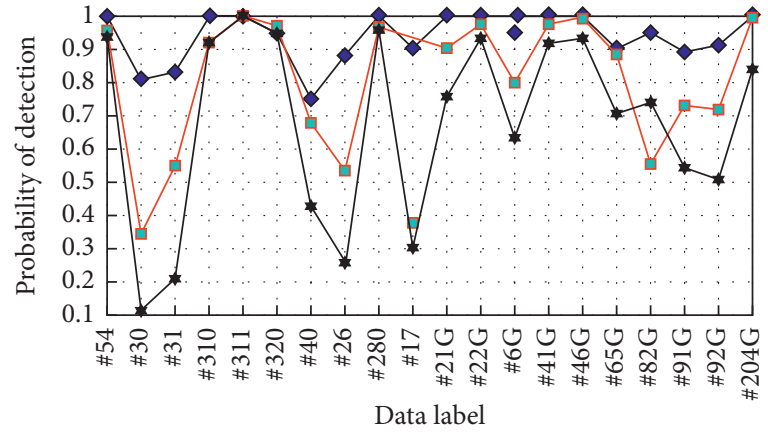

Proposed cICA-based detector with CCEBM

$\square-$ Feature-compression-based detector [19]

* Adaptive composite GLRT detector [26]

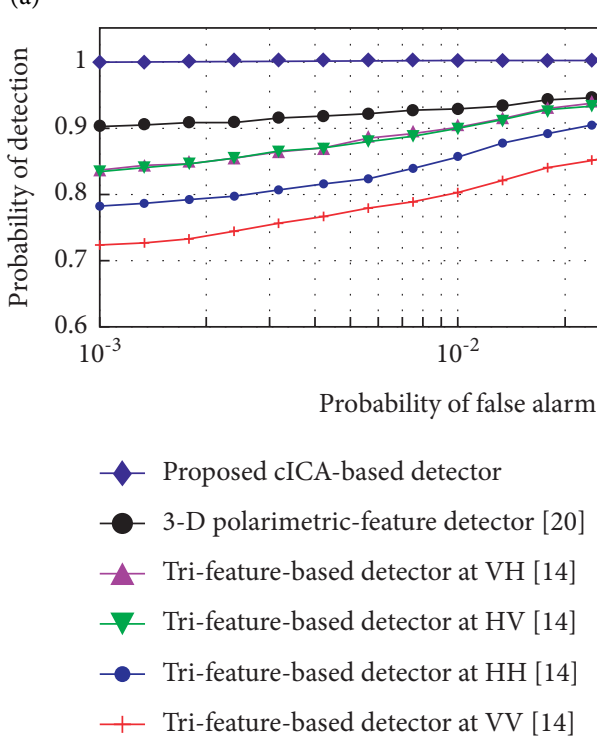

(b)

(c)

Figure 6: The probabilities of detection for all twenty datasets at VV polarization: (a) proposed detector, feature-based detector using three TF features [15], trifeature-based detector [14], and fractal-based detector [10, 11]; (b) proposed detector, feature-compression-based detector [19], and adaptive composite GLRT detector [24]; (c) ROC curves for the proposed detector at VV polarization, 3D polarimetricfeature detector [19], and trifeature-based detector [14] at $\mathrm{HH}, \mathrm{HV}, \mathrm{VH}$, and $\mathrm{VV}$, while $T=512, T_{\mathrm{Ref}}=0$, and $T_{\mathrm{CPI}}=512$.

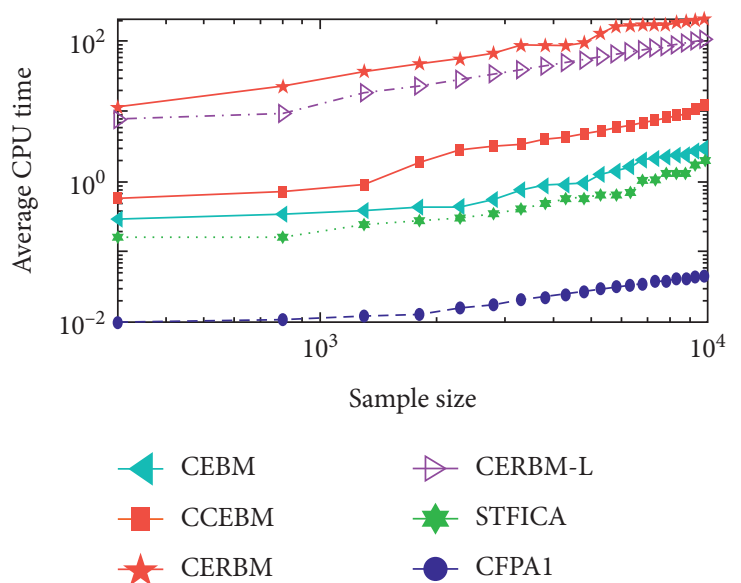

(a)
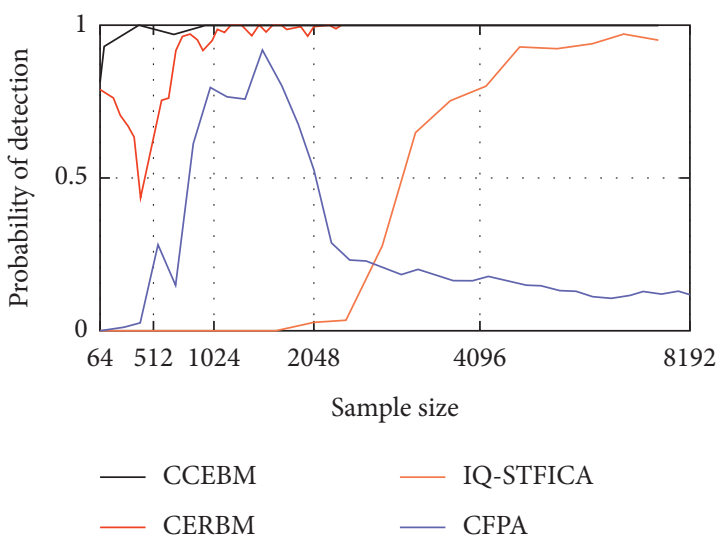

(b)

FIgURE 7: (a) Computational time of the proposed CCEBM against other cICA algorithms: (b) probability of detection of the cICA-based detector versus the sample size of the cICA block input mixtures where the SCR is equal to SCR $=-5$ for Tg-SW 2 . 


\section{Conclusion}

Herein, we first established a new convolutive complex-valued ICA algorithm, which is an extension of the complex-valued entropy bound minimization (CEBM), to separate and detect targets in maritime radars. Then, a new cICA-based detector with an open structure was proposed to detect targets in practical scenarios. This detector makes the detection decision through four distinct blocks. The experimental results on reallife IPIX radar data with injected synthetic targets were presented to demonstrate the superior separation performance and computational efficiency of the CCEBM algorithm. The detection performance of the cICA-based detector was also presented and analyzed. An extensive detection performance analysis and comparison was conducted when the detector utilized CCEBM, CERBM, or IQ-STFICA. CCEBM attained the best separation performance followed by CERBM and IQSTFICA, respectively. The detector obtained the highest detection probabilities with CCEBM. It managed to detect targets with nonlinear Doppler modulation without requiring a specific motion model. The proposed detector is based on nonenergy characteristics, and this reduces its dependence on SCR, as depicted by experiments. The experiments revealed the robustness of the detector since its detection performance was approximately the same in high and low sea states. Promising results with the injected synthetic Swerling target model in different sea states were obtained. In conclusion, the experiments on different polarizations of the real-life IPIX radar data for detecting real-life sea-surface small floating targets manifested the superiority of the detector over several novel detectors.

\section{Data Availability}

No data were used to support this study.

\section{Conflicts of Interest}

The authors declare that they have no conflicts of interest.

\section{References}

[1] X.-L. Li and T. Adali, "Complex independent component analysis by entropy bound minimization," IEEE Transactions on Circuits and Systems I: Regular Papers, vol. 57, no. 7, pp. 1417-1430, 2010.

[2] H. Ghahramani, N. Parhizgar, B. A. Arand, and M. Barari, "Ground (Weibull-distributed) clutter suppression based on independent component analysis for detection of swerling target models," Journal of Communications Technology and Electronics, vol. 65, no. 2, pp. 160-171, 2020.

[3] M. Novey and T. Adali, "Using complex-valued ICA to efficiently combine radar polarimetric data for target detection," in Proceedings of the 2009 IEEE International Conference on Acoustics, Speech and Signal Processing, pp. 1673-1676, Taipei, Taiwan, 2009.

[4] H. Ghahramani, M. Barari, and M. H. Bastani, "Maritime radar target detection in presence of strong sea clutter based on blind source separation," IETE Journal of Research, vol. 60, no. 5, pp. 331-344, 2014.
[5] H. Ghahramani, M. Barari, and M. H. Bastani, "Suppressing sea clutter in maritime radar based on DUET BSS," Journal of Iranian Association of Electrical and Electronics Engineers, vol. 12, no. 2, 2015.

[6] R. Vicen-bueno, M. Rosa-zurera, M. P. Jarabo-Amores, D. de la Mata-Moya, and D. De Mata-moya, "Coherent detection of swerling 0 targets in sea-ice Weibull-distributed clutter using neural networks," IEEE Transactions on Instrumentation and Measurement, vol. 59, no. 12, pp. 31393151, 2010.

[7] Y. Xu, C. Hou, S. Yan, J. Li, and C. Hao, "Fuzzy statistical normalization CFAR detector for non-Rayleigh data," IEEE Transactions on Aerospace and Electronic Systems, vol. 51, no. 1, pp. 383-396, 2015.

[8] K. Yan, Y. Bai, H.-C. Wu, and X. Zhang, "Robust target detection within sea clutter based on graphs," IEEE Transactions on Geoscience and Remote Sensing, vol. 57, no. 9, pp. 7093-7103, 2019.

[9] Y. Li, P. Xie, Z. Tang, T. Jiang, and P. Qi, "SVM-based seasurface small target detection: a false-alarm-rate-controllable approach," IEEE Geoscience and Remote Sensing Letters, vol. 16, no. 8, pp. 1225-1229, 2019.

[10] J. Hu, W.-W. Tung, and J. Gao, "Detection of low observable targets within sea clutter by structure function based multifractal analysis," IEEE Transactions on Antennas and Propagation, vol. 54, no. 1, pp. 136-143, 2006.

[11] F. Luo, D. Zhang, and B. Zhang, "The fractal properties of sea clutter and their applications in maritime target detection," IEEE Geoscience and Remote Sensing Letters, vol. 10, no. 6, pp. 1295-1299, 2013.

[12] X.-K. Xu, "Low observable targets detection by joint fractal properties of sea clutter: an experimental study of IPIX OHGR datasets," IEEE Transactions on Antennas and Propagation, vol. 58, no. 4, pp. 1425-1429, 2010.

[13] P.-L. Shui, D.-C. Li, and S.-W. Xu, "Tri-feature-based detection of floating small targets in sea clutter," IEEE Transactions on Aerospace and Electronic Systems, vol. 50, no. 2, pp. 1416-1430, 2014.

[14] S.-N. Shi and P.-L. Shui, "Sea-surface floating small target detection by one-class classifier in time-frequency feature space," IEEE Transactions on Geoscience and Remote Sensing, vol. 56, no. 11, pp. 6395-6411, 2018.

[15] C. Feng, H. Zheng, L. Zhou, and J. Cheng, "Detection performance analysis of recurrence quantification analysis measures for low observable target within sea clutter under different sea conditions," IET Radar, Sonar \& Navigation, vol. 9, no. 4, pp. 447-456, 2015.

[16] Y. Shi, X. Xie, and D. Li, "Range distributed floating target detection in sea clutter via feature-based detector," IEEE Geoscience and Remote Sensing Letters, vol. 13, no. 12, pp. 1847-1850, 2016.

[17] D. Li and P. Shui, "Floating small target detection in sea clutter via normalised Hurst exponent," Electronics Letters, vol. 50, no. 17, pp. 1240-1242, 2014.

[18] P. Shui, Z. Guo, and S. Shi, "Feature-compression-based detection of sea-surface small targets," IEEE Access, vol. 8, pp. 8371-8385, 2020.

[19] S. Xu, J. Zheng, J. Pu, and P. Shui, "Sea-surface floating small target detection based on polarization features," IEEE Geoscience and Remote Sensing Letters, vol. 15, no. 10, pp. 1-5, 2018.

[20] X. Chen, J. Guan, Z. Bao, and Y. He, "Detection and extraction of target with micromotion in spiky sea clutter via short-time 
fractional Fourier transform," IEEE Transactions on Geoscience and Remote Sensing, vol. 52, no. 2, pp. 1002-1018, 2014.

[21] H. Xing and Y. Yan, "Detection of low-flying target under the sea clutter background based on Volterra filter," Complexity, vol. 2018, Article ID 1513591, 12 pages, 2018.

[22] J. Nie, Y. Xiao, L. Huang, and F. Lv, "Time-frequency analysis and target recognition of HRRP based on CN-LSGAN, STFT, and CNN," Complexity, vol. 2021, Article ID 6664530, 10 pages, 2021.

[23] A. De Maio and M. Greco, Modern Radar Detection Theory, Institution of Engineering and Technology, London, UK, 2015.

[24] J. Cai, H. Zhou, W. Huang, and B. Wen, "Ship detection and direction finding based on time-frequency analysis for compact HF radar," IEEE Geoscience and Remote Sensing Letters, vol. 18, no. 1, pp. 72-76, 2021.

[25] IPIX Radar, "The McMaster IPIX radar sea clutter database," 2001, http://soma.ece.mcmaster.ca/ipix/.

[26] A. Hyvärinen, J. Karhunen, and E. Oja, Independent Component Analysis, John Wiley \& Sons, Hoboken, NJ, USA, 2001.

[27] Y. Xiang, D. Peng, and Z. Yang, "Dependent component analysis using precoding," Blind Source Separation, Elsevier, Amsterdam, Netherlands, pp. 73-90, 2015.

[28] E. Moreau and T. Adali, Blind Identification and Separation of Complex-Valued Signals, Wiley, Hoboken, NJ, USA, 2013.

[29] S. C. Douglas, M. Gupta, H. Sawada, and S. Makino, "Spatio-temporal fast ICA algorithms for the blind separation of convolutive mixtures," IEEE Transactions on Audio, Speech and Language Processing, vol. 15, no. 5, pp. 1511-1520, 2007.

[30] G.-S. Fu, R. Phlypo, M. Anderson, and T. Adal, "Complex independent component analysis using three types of diversity: non-Gaussianity, nonwhiteness, and noncircularity," IEEE Transactions on Signal Processing, vol. 63, no. 3, pp. 794-805, 2015.

[31] M. Novey and T. Adali, "On extending the complex fast ICA algorithm to noncircular sources," IEEE Transactions on Signal Processing, vol. 56, no. 5, pp. 2148-2154, 2008.

[32] H. Ghahramani, N. Parhizgar, B. A. Arand, and M. Barari, "Polarimetric detection of maritime floating small target based on the complex-valued entropy rate bound minimization," Heliyon, vol. 6, no. 10, Article ID e05138, 2020.

[33] S. Haykin, Adaptive Radar Signal Processing, John Wiley \& Sons, Hoboken, NJ, USA, 2007.

[34] X.-L. Li and T. Adali, "A novel entropy estimator and its application to ICA," in Proceedings of the 2009 IEEE International Workshop on Machine Learning for Signal Processing, Grenoble, France, 2009.

[35] S.-N. Shi and P.-L. Shui, "Detection of low-velocity and floating small targets in sea clutter via income-reference particle filters,” Signal Processing, vol. 148, pp. 78-90, 2018.

[36] X. Chen, W. Huang, C. Zhao, and Y. Tian, "Rain detection from X-band marine radar images: a support vector machinebased approach," IEEE Transactions on Geoscience and Remote Sensing, vol. 58, no. 3, pp. 2115-2123, 2020.

[37] X. Chen and W. Huang, "Identification of rain and lowbackscatter regions in X-band marine radar images: an unsupervised approach," IEEE Transactions on Geoscience and Remote Sensing, vol. 58, no. 6, pp. 4225-4236, 2020.

[38] A. K. Mishra, "Validation of PCA and LDA for SAR ATR," in Proceedings of the 2008 IEEE Region 10 Conference, Hyderabad, India, 2008. 